\title{
Lowest Weight Representations of Some Infinite Dimensional Groups on Fock Spaces
}

\author{
MARTIN U. SCHMIDT \\ Institut für Theoretische Physik, Freie Universität Berlin, Berlin, West Germany
}

(Received: 7 February 1989; in final form: 12 December 1989)

\begin{abstract}
The results of Kashiwara and Vergne on the decomposition of the tensor products of the 'SegalShale-Weil representation' are extended to the infinite dimensional case and give all unitary lowest weight representations. Our methods are basically algebraic. When restricted to the finite dimensional case, they yield a new proof.
\end{abstract}

AMS subject classifications (1980). 22E65, 22E70.

Key words. Highest weight modules, CCR algebras, representation theory.

\section{The Representations of $\mathrm{U}(V, W)$}

If $V$ and $W$ are two separable Hilbert spaces, $\mathrm{U}(V, W)$ is defined to be subgroup of $\mathrm{GL}(V \oplus W)$, the group of all invertible bounded operators of the Hilbert space $V \oplus W$, which leaves the Hermitian form defined by the operator

$$
J=\left(\begin{array}{rr}
1 & 0 \\
0 & -1
\end{array}\right)
$$

on $V \oplus W$ invariant.

Thus $A \in \mathrm{U}(V, W)$, if and only if $J A^{*-1} J^{-1}=A$. With respect to the decomposition $V \oplus W$ let $A$ be expressed as

$$
A=\left(\begin{array}{ll}
a & b \\
c & d
\end{array}\right)
$$

Then $A \in \mathrm{U}(V, W)$ if and only if

$$
\begin{array}{ll}
a a^{*}-b b^{*}=1 & a^{*} a-c^{*} c=1, \\
d d^{*}-c c^{*}=1 & d^{*} d-b^{*} b=1, \\
a c^{*}-b d^{*}=0 & a^{*} b-c^{*} d=0 .
\end{array}
$$

In particular, $a$ and $d$ are invertible operators. Therefore, $A$ can be expressed as a product

$$
\left(\begin{array}{ll}
a & b \\
c & d
\end{array}\right)=\left(\begin{array}{cc}
1 & b d^{-1} \\
0 & 1
\end{array}\right)\left(\begin{array}{cc}
a-b d^{-1} c & 0 \\
0 & d
\end{array}\right)\left(\begin{array}{cc}
1 & 0 \\
d^{-1} c & 1
\end{array}\right)
$$


Let $U_{\text {res }}(V, W)$ be the subgroup of $\mathrm{U}(V, W)$ consisting of these elements

$$
\left(\begin{array}{ll}
a & b \\
c & d
\end{array}\right)
$$

for which $b: W \rightarrow V$ and $c: V \rightarrow W$ are Hilbert-Schmidt operators. Since the HilbertSchmidt operators form a two-sided ideal in the space of the bounded operators, $\mathrm{U}_{\text {res }}(V, W)$ is a subgroup of $\mathrm{U}(V, W)$. Now

$$
d^{-1} c c^{*} d^{*-1}=1-d^{-1} d^{*-1} \text { and } d^{*-1} b^{*} b d^{-1}=1-d^{*-1} d^{-1}
$$

Therefore, two inequalities must hold

$$
\left\|d^{-1} c\right\|<1 \text { and }\left\|b d^{-1}\right\|<1 .
$$

Consider two elements $A_{1}$ and $A_{2}$ of $\mathrm{U}_{\text {res }}(V, W)$ and the third element $A_{3}=A_{1} A_{2}$ with

$$
A_{i}=\left(\begin{array}{ll}
a_{i} & b_{i} \\
c_{i} & d_{i}
\end{array}\right), \quad i=1,2,3 .
$$

Then $d_{1}^{-1} d_{3} d_{2}^{-1}=1+d_{1}^{-1} c_{1} b_{2} d_{2}^{-1}$ is of the form $1+$ traceclass, which guarantees the determinant of $d_{1}^{-1} d_{3} d_{2}^{-1}$ to be defined. The inequalities $\left\|d_{1}^{-1} c_{1}\right\|<1$ and $\left\|b_{2} d_{2}^{-1}\right\|<1$ show that $\operatorname{det}\left(d_{1}^{-1} d_{3} d_{2}^{-1}\right)$ cannot be zero.

Then $c\left(A_{1}, A_{2}\right):=\operatorname{det}^{-1}\left(d_{1}^{-1} d_{3} d_{2}^{-1}\right)$ is a cocycle, which induces a central extension

$$
\tilde{\mathrm{U}}_{\mathrm{res}}(V, W):=\left\{\left(\left(\begin{array}{ll}
a & b \\
c & d
\end{array}\right), z\right), \text { with } z \bar{z}=\operatorname{det}\left(1-d^{*-1} b^{*} b d^{-1}\right)\right\}
$$

of $\mathrm{U}_{\text {res }}(V, W)$. Indeed by direct calculation one shows

$$
c\left(A_{1}, A_{2}\right) c\left(A_{1} A_{2}, A_{3}\right)=c\left(A_{1}, A_{2} A_{3}\right) c\left(A_{2}, A_{3}\right)
$$

for arbitrary elements $A_{1}, A_{2}, A_{3}$ of $\mathrm{U}_{\text {res }}(V, W)$.

1.1. Remark. If $W$ is finite dimensional, the cocycle is induced by the function

$$
\left(\begin{array}{ll}
a & b \\
c & d
\end{array}\right) \rightarrow \operatorname{det}^{-1}(d)
$$

on $\mathrm{U}(V, W)$ and, therefore, is trivial. Due to the symmetry of $\mathrm{U}(V, W)$ in $V$ and $W$ the same is true, if $V$ is finite dimensional. So in these cases it is not necessary to consider central extensions of $\mathrm{U}(V, W)$.

The subject of this section is the discussion of the unitary lowest weight representations of $\tilde{\mathrm{U}}_{\text {res }}(V, W)$, namely the irreducible components of the $k$-fold tensor product of the so called 'Segal-Shale-Weil representations' [1]. In the finite dimensional case, all results are known, however, not with those algebraic methods which will be used in this note.

In the infinite dimensional case, we first define a representation of the corresponding Lie algebra and then we show that this representation may be lifted to a representation of the group. 
For any Hilbert space $H$ let $L(H)$ denote the Lie algebra of bounded operators of $H$ and $C(H)$ the subalgebra of all compact operators of $H$. Let $L_{\text {res }}(V, W)$ denote the subalgebra of $L V \oplus W)$, which consists of elements of the form

$$
\left(\begin{array}{ll}
a & b \\
c & d
\end{array}\right), \quad b: W \rightarrow V, c: V \rightarrow W,
$$

$b$ and $c$ being Hilbert-Schmidt operators. Let $A_{1}$ and $A_{2}$ be two elements of $L_{\text {res }}(V, W)$, written again as

$$
A_{1}=\left(\begin{array}{ll}
a_{1} & b_{1} \\
c_{1} & d_{1}
\end{array}\right), \quad A_{2}=\left(\begin{array}{ll}
a_{2} & b_{2} \\
c_{2} & d_{2}
\end{array}\right)
$$

The cocycle $\gamma\left(A_{1}, A_{2}\right):=\operatorname{tr}\left(c_{2} b_{1}-c_{1} b_{2}\right)$ induces a central extension $\tilde{L}_{\mathrm{res}}(V, W)$ of $L_{\text {res }}(V, W)$.

1.2. Remark. On the Lie algebra level it is obvious, that if either $V$ or $W$ are finite dimensional then the cocycle $\gamma$ is a boundary and therefore trivial. Indeed, if $V$ is finite dimensional, it is the boundary of the functional

$$
\left(\begin{array}{ll}
a & b \\
c & d
\end{array}\right) \rightarrow \operatorname{tr}(a)
$$

and if $W$ is finite dimensional, it is the boundary of the functional

$$
\left(\begin{array}{ll}
a & b \\
c & d
\end{array}\right) \rightarrow-\operatorname{tr}(d)
$$

because $\operatorname{tr}\left(b_{1} c_{2}-b_{2} c_{1}\right)=-\operatorname{tr}\left(c_{1} b_{2}-c_{2} b_{1}\right)$ holds and the trace of a commutant is zero.

The 'Segal-Shale-Weil representation' is in some sense the transformation of the canonical representation of the Lie algebra $L(H)$ for some Hilbert space $H$ on the vector space of the symmetric algebra over this Hilbert space. So let us first consider this representation.

Let $H$ be a separable Hilbert space. Let $S^{n}(H)$ denote the Hilbert space completion of the vector space of $n$-powers of the symmetric algebra of $H$ with the Hermitian form:

$$
\left\langle h_{1} h_{2} \cdots h_{n}, h_{1}^{\prime} h_{2}^{\prime} \cdots h_{n}^{\prime}\right\rangle=\sum_{\sigma} \prod_{i=1}^{n}\left\langle h_{\sigma(i)}, h_{i}^{\prime}\right\rangle,
$$

where the sum is over all permutations $\sigma$ of $(1,2, \ldots, n)$.

Define $\check{S}(H):=\bigoplus_{n \in \mathbb{N}} S^{n}(H)$ endowed with the final topology defined by the inclusions $i_{n}: S^{n}(H) \rightarrow \breve{S}(H)$. Let $S(H)$ be the Hilbert space completion of $\breve{S}(H)$. Let $\hat{S}(H)$ be $\Pi_{n} S^{n}(H)$ with the initial topology defined by the projections $p_{n}: \hat{S}(H) \rightarrow S^{n}(H) . \hat{S}(H)$ and $\breve{S}(H)$ are the antilinear dual spaces of each other and $\check{S}(H) \subseteq S(H) \subseteq \widehat{S}(H)$ with continuous and dense inclusions. 
For any $h \in H$ let $a^{*}(h): \hat{S}(H) \rightarrow \hat{S}(H)$ denote the continuous operator of multiplication with $h$. This operator is, of course, also a continuous operator on $\breve{S}(H)$. So the adjoint of this operator $a(h)$ is a continuous operator on both $\hat{S}(H)$ and $\breve{S}(H)$. It is well known that they obey the following commutation relations

$$
\begin{aligned}
& {\left[a^{*}(h), a^{*}\left(h^{\prime}\right)\right]=0=\left[a(h), a\left(h^{\prime}\right)\right]} \\
& {\left[a(h), a^{*}\left(h^{\prime}\right)\right]=\left\langle h, h^{\prime}\right\rangle 1 .}
\end{aligned}
$$

The operators $a^{*}(h)$ and $a(h)$ can be used to construct the canonical representation of $L(H)$ on $\hat{S}(H)$. Let $\left\{h_{i}\right\}_{i \in \mathbb{N}}$ be any orthonormal basis of $H$. For any operator $a \in L(H)$ set

$$
\mathrm{d} \Gamma(a):=\sum_{i, j} a^{*}\left(h_{i}\right) a\left(h_{j}\right)\left\langle h_{i}, a h_{j}\right\rangle .
$$

This sum converges only pointwise. $\mathrm{d} \Gamma(a)$ does not depend on the choice of the orthonormal basis, because $a^{*}(h)$ is linear in $h$ and $a(h)$ is antilinear in $h . \mathrm{d} \Gamma(a)$ maps $S^{n}(H)$ into $S^{n}(H)$ and the norm of this map is smaller than $n\|a\|$. Therefore, $\mathrm{d} \Gamma(a)$ is continuous on $\hat{S}(H)$ and $\breve{S}(H)$.

Let us now construct the representation of $\tilde{L}_{\text {res }}(V, W)$. We denote by $\bar{W}$ the corresponding antilinear Hilbert space of $W$ and for all $w \in W$ by $\bar{w}$ the corresponding element of $\bar{W}$. Choose two orthonormal basis $\left\{v_{i}\right\}_{i \in \mathbb{N}}$ and $\left\{w_{i}\right\}_{i \in \mathbb{N}}$ of $V$ and $W$ respectively. Now a representation $\mathrm{d} \tilde{\Gamma}$ of $\tilde{L}_{\text {res }}(V, W)$ on $\hat{S}(V \oplus \bar{W})$ and $\breve{S}(V \oplus \bar{W})$ respectively is defined by

$$
\begin{aligned}
\mathrm{d} \tilde{\Gamma} & \left(\left(\begin{array}{ll}
a & b \\
c & d
\end{array}\right), z\right) \\
:= & \sum_{i, j} a^{*}\left(v_{i}\right) a\left(v_{j}\right)\left\langle v_{i}, a v_{j}\right\rangle- \\
& -\sum_{i, j} a^{*}\left(\bar{w}_{i}\right) a\left(\bar{w}_{j}\right)\left\langle w_{j}, d w_{i}\right\rangle+ \\
& +\sum_{i, j} a^{*}\left(\bar{w}_{i}\right) a^{*}\left(v_{j}\right)\left\langle v_{j}, b w_{i}\right\rangle- \\
& -\sum_{i, j} a\left(v_{i}\right) a\left(\bar{w}_{j}\right)\left\langle w_{j}, c v_{i}\right\rangle+z \quad 1 .
\end{aligned}
$$

This sum converges only pointwise. $a$ and $d$ are bounded operators, hence

$$
\mathrm{d} \tilde{\Gamma}\left(\left(\begin{array}{ll}
a & 0 \\
0 & d
\end{array}\right), z\right)
$$

is continuous. $b$ and $c$ are Hilbert-Schmidt operators, hence

$$
\mathrm{d} \tilde{\Gamma}\left(\left(\begin{array}{ll}
0 & b \\
c & 0
\end{array}\right), z\right)
$$

is continuous. Because of the linearity of $a^{*}(v)$ and $a(\bar{w})$ and the antilinearity of $a(v)$ and $a^{*}(\bar{w})$ in $v$ and $w$, respectively, $\mathrm{d} \tilde{\Gamma}$ does not depend on the choice of the orthonormal 
basis. For all Hilbert-Schmidt operators $b: W \rightarrow V$ we will also denote the element $\Sigma_{i, j} v_{i} \otimes \bar{w}_{j}\left\langle v_{i}, b w_{j}\right\rangle$ of $S^{1}(V) \otimes S^{1}(\bar{W})$ by $b$. Then

$$
\left(\left(\begin{array}{ll}
0 & b \\
0 & 0
\end{array}\right), 0\right)
$$

is represented by the operator of multiplication with $b$ and

$$
\left(\left(\begin{array}{ll}
0 & 0 \\
c & 0
\end{array}\right), 0\right)
$$

is represented by the adjoint operator of multiplication with $-c^{*}$. The diagonal elements

$$
\left(\left(\begin{array}{ll}
a & 0 \\
0 & d
\end{array}\right), z\right)
$$

are represented by the canonical representation of $a$ minus the canonical representation of $d^{t}$ plus $z$ times the identity. If $b$ and $c$ are finite rank operators direct calculation shows $\mathrm{d} \tilde{\Gamma}$ to be a representation. By continuity, it follows that $\mathrm{d} \tilde{\Gamma}$ is a representation of $\tilde{L}_{\text {res }}(V, W)$ (see A. Carey and S. Ruijsenaars [2]).

1.3. Remark. This representation could be thought of as the transformed canonical representation of $\tilde{L}_{\mathrm{res}}(V, W)$ on $\hat{S}(V \oplus W)$. However, the transformation:

$$
a^{*}(w) \rightarrow-a(\bar{w}) \quad \text { and } \quad a(w) \rightarrow a^{*}(\bar{w})
$$

is not defined on elements of the form

$$
\left(\begin{array}{ll}
0 & 0 \\
0 & d
\end{array}\right)
$$

of $L_{\text {res }}(V, W)$, if $d$ is not of trace-class. Thus renormalisation makes it necessary to consider the central extension $\tilde{L}_{\text {res }}(V, W)$ of $L_{\text {res }}(V, W)$.

We will prove now that $\mathrm{d} \tilde{\Gamma}$ can be lifted to a representation of $\tilde{U}_{\text {res }}(V, W)$. The elements of this group can be thought of as linear symmetries of the free Boson fields over $V \oplus \bar{W}$. Under this point of view, the question arises which symmetries can be implemented into a given representation of the canonical commutation relations (CCR) corresponding to this free Boson fields. There is an extensive literature on this question (see, for example, [3-5]). For all bounded operators

$$
\left(\begin{array}{ll}
a & b \\
c & d
\end{array}\right) \text { on } V \oplus W
$$

whose component $d$ is invertible and for all $z \in U(\mathbb{C})$, the product decomposition

$$
\left(\begin{array}{cc}
1 & b d^{-1} \\
0 & 1
\end{array}\right)\left(\begin{array}{cc}
a-b d^{-1} c & 0 \\
0 & d
\end{array}\right)\left(\begin{array}{cc}
1 & 0 \\
d^{-1} c & 1
\end{array}\right)
$$


determines completely the representation map

$$
\begin{aligned}
& \tilde{\Gamma}\left(\left(\begin{array}{ll}
a & b \\
c & d
\end{array}\right), z\right) \\
& \quad:=\exp \left(\mathrm{d} \tilde{\Gamma}\left(\left(\begin{array}{cc}
0 & b d^{-1} \\
0 & 0
\end{array}\right), 0\right)\right) \tilde{\Gamma}\left(\left(\begin{array}{cc}
a-b d^{-1} c & 0 \\
0 & d
\end{array}\right), z\right) \exp \left(\mathrm{d} \tilde{\Gamma}\left(\left(\begin{array}{cc}
0 & 0 \\
d^{-1} c & 0
\end{array}\right), 0\right) .\right.
\end{aligned}
$$

The middle component is defined to be the canonical representation of $a-b d^{-1} c$ times the canonical representation of $d^{t-1}$ times $z$.

$$
\tilde{\Gamma}\left(\left(\begin{array}{ll}
1 & 0 \\
c & 1
\end{array}\right), 1\right)
$$

is a continuous operator on $\breve{S}(V \oplus \bar{W})$, which intertwines with $\mathrm{d} \tilde{\Gamma}$ by

$$
\begin{aligned}
& \tilde{\Gamma}\left(\left(\begin{array}{ll}
1 & 0 \\
c & 1
\end{array}\right), 1\right) \mathrm{d} \tilde{\Gamma}\left(\left(\begin{array}{ll}
a^{\prime} & b^{\prime} \\
c^{\prime} & d^{\prime}
\end{array}\right), z^{\prime}\right) \\
& \quad=\mathrm{d} \tilde{\Gamma}\left(\left(\begin{array}{ll}
1 & 0 \\
c & 1
\end{array}\right)\left(\begin{array}{ll}
a^{\prime} & b^{\prime} \\
c^{\prime} & d^{\prime}
\end{array}\right)\left(\begin{array}{rr}
1 & 0 \\
-c & 1
\end{array}\right), z^{\prime}-\operatorname{tr}\left(c b^{\prime}\right)\right) \tilde{\Gamma}\left(\left(\begin{array}{ll}
1 & 0 \\
c & 1
\end{array}\right), 1\right) .
\end{aligned}
$$

Note that

$$
\tilde{\Gamma}\left(\left(\begin{array}{ll}
a & 0 \\
0 & d
\end{array}\right), z\right)
$$

is a continuous operator on both $\check{S}(V \oplus \bar{W})$ and $\hat{S}(V \oplus \bar{W})$, which intertwines with $\mathrm{d} \tilde{\Gamma}$ by

$$
\begin{aligned}
& \tilde{\Gamma}\left(\left(\begin{array}{ll}
a & 0 \\
0 & d
\end{array}\right), z\right) \mathrm{d} \tilde{\Gamma}\left(\left(\begin{array}{ll}
a^{\prime} & b^{\prime} \\
c^{\prime} & d^{\prime}
\end{array}\right), z^{\prime}\right) \\
& \quad=\mathrm{d} \tilde{\Gamma}\left(\left(\begin{array}{ll}
a & 0 \\
0 & d
\end{array}\right)\left(\begin{array}{ll}
a^{\prime} & b^{\prime} \\
c^{\prime} & d^{\prime}
\end{array}\right)\left(\begin{array}{cc}
a^{-1} & 0 \\
0 & d^{-1}
\end{array}\right), z^{\prime}\right) \tilde{\Gamma}\left(\left(\begin{array}{ll}
a & 0 \\
0 & d
\end{array}\right), z\right) .
\end{aligned}
$$

Here

$$
\tilde{\Gamma}\left(\left(\begin{array}{ll}
0 & b \\
0 & 0
\end{array}\right), 1\right)
$$

is a continuous operator on $\hat{S}(V \oplus \bar{W})$, which intertwines with $\mathrm{d} \tilde{\Gamma}$ by

$$
\begin{aligned}
& \tilde{\Gamma}\left(\left(\begin{array}{ll}
1 & b \\
0 & 1
\end{array}\right), 1\right) \mathrm{d} \tilde{\Gamma}\left(\left(\begin{array}{ll}
a^{\prime} & b^{\prime} \\
c^{\prime} & d^{\prime}
\end{array}\right), z^{\prime}\right) \\
& \quad=\mathrm{d} \tilde{\Gamma}\left(\left(\begin{array}{ll}
1 & b \\
0 & 1
\end{array}\right)\left(\begin{array}{ll}
a^{\prime} & b^{\prime} \\
c^{\prime} & d^{\prime}
\end{array}\right)\left(\begin{array}{cc}
1 & -b \\
0 & 1
\end{array}\right), z^{\prime}+\operatorname{tr}\left(c^{\prime} b\right)\right) \mathrm{d} \tilde{\Gamma}\left(\left(\begin{array}{ll}
1 & b \\
0 & 1
\end{array}\right), 1\right) .
\end{aligned}
$$




\subsection{LEMMA}

$$
\exp \left(\mathrm{d} \tilde{\Gamma}\left(\left(\begin{array}{ll}
0 & b \\
0 & 0
\end{array}\right), 0\right)\right)
$$

is a continuous operator from $\check{S}(V \oplus \bar{W})$ into $S(V \oplus \bar{W})$ if $\|b\|<1$.

$$
\exp \left(d \tilde{\Gamma}\left(\left(\begin{array}{ll}
0 & 0 \\
c & 0
\end{array}\right), 0\right)\right)
$$

is a continuous operator from $S(V \oplus \bar{W})$ into $\hat{S}(V \oplus \bar{W})$, if $\|c\|<1$.

Proof. Let $x \in S^{p}(V) \otimes S^{q}(\bar{W})$ and $x^{\prime} \in S^{p^{\prime}}(V) \otimes S^{q^{\prime}}(\bar{W})$ be two elements of $S(V \oplus \bar{W})$. By definition of the Hermitian product it follows that

$$
\left\langle x x^{\prime}, x x^{\prime}\right\rangle \leqslant\left(\begin{array}{c}
p+p^{\prime} \\
p
\end{array}\right)\left(\begin{array}{c}
q+q^{\prime} \\
q
\end{array}\right)\langle x, x\rangle\left\langle x^{\prime}, x^{\prime}\right\rangle .
$$

For all Hilbert-Schmidt operators $b: W \rightarrow V$ let $b$ also denote the element $\Sigma_{i, j} v_{i} \otimes \bar{w}_{j}\left\langle v_{i}, b w_{j}\right\rangle$ of $S(V \oplus \bar{W})$. The inequality above implies

$$
\left\langle b^{n} x, b^{n} x\right\rangle \leqslant\left(\begin{array}{c}
p+n \\
n
\end{array}\right)\left(\begin{array}{c}
q+n \\
n
\end{array}\right)\langle x, x\rangle\left\langle b^{n}, b^{n}\right\rangle .
$$

Let $p_{n}\left(b^{*} b\right)$ be the sum of the $n$th powers of the eigenvalues of $b^{*} b, p_{n}\left(b^{*} b\right)=\operatorname{tr}\left(\left(b^{*} b\right)^{n}\right)$ and for each partition $\lambda p_{\lambda}\left(b^{*} b\right)$, the corresponding polynomial in such power sums (see Macdonald [6]). By a combinatorial calculation $(1 / n !)^{2}\left\langle b^{n}, b^{n}\right\rangle$ is equal to

$$
\sum_{|\lambda|=n} z_{\lambda}^{-1} p_{\lambda}\left(b^{*} b\right)=h_{n}\left(b^{*} b\right)
$$

the $n$th complete symmetric function in the eigenvalues of $b^{*} b$. Set $\beta$ for

$$
\frac{1}{\|b\|^{2}} \operatorname{tr}\left(b^{*} b\right)
$$

Then the inequality $p_{n}\left(b^{*} b\right) \leqslant \beta\|b\|^{2 n}$ is obvious. This implies

$$
h_{n}\left(b^{*} b\right) \leqslant\left(\begin{array}{c}
\beta+n \\
n
\end{array}\right)\|b\|^{2 n}
$$

and, finally,

$$
\begin{aligned}
\langle\exp (b) x, \exp (b) x\rangle & \leqslant \sum_{n}\left(\begin{array}{c}
p+n \\
n
\end{array}\right)\left(\begin{array}{c}
q+n \\
n
\end{array}\right)\left(\begin{array}{c}
\beta+n \\
n
\end{array}\right)\|b\|^{2 n}\langle x, x\rangle \\
& \leqslant\left(\begin{array}{c}
p+q \\
p
\end{array}\right)\left(\begin{array}{c}
p+q+\beta \\
p+q
\end{array}\right) \sum_{n}\left(\begin{array}{c}
p+q+\beta+n \\
n
\end{array}\right)\|b\|^{2 n}\langle x, x\rangle \\
& \leqslant\left(\begin{array}{c}
p+q \\
p
\end{array}\right)\left(\begin{array}{c}
p+q+\beta \\
p+q
\end{array}\right)\left(\frac{1}{1-\|b\|^{2}}\right)^{p+q+\beta}\langle x, x\rangle .
\end{aligned}
$$


This shows the first part of the lemma. The operators of the second part are just the adjoint operators of the operators in the first part. This completes the proof.

Now in the product decomposition of $\tilde{\Gamma}$ the first factor can either be an operator of $\check{S}(V \oplus \bar{W})$ or an operator from $S(V \oplus \bar{W})$ into $\hat{S}(V \oplus \bar{W})$, the second factor can be an operator of $\breve{S}(V \oplus \bar{W})$ or an operator of $\breve{S}(V \oplus \bar{W})$, and the third factor can be an operator from $\breve{S}(V \oplus \bar{W})$ into $S(V \oplus \bar{W})$ or an operator of $\hat{S}(V \oplus \bar{W})$. Thus, the image of $\tilde{\Gamma}$ consists either of continuous operators from $\check{S}(V \oplus \bar{W})$ into $S(V \oplus \bar{W})$ or continuous operators from $S(V \oplus \bar{W})$ into $\hat{S}(V \oplus \bar{W})$ or, of course, of continuous operators from $\breve{S}(V \oplus \bar{W})$ into $\hat{S}(V \oplus \bar{W})$. In the following, the actual intended meaning should be clear from the context.

1.5. LEMMA. The composition of two such operators is compatible with the group multiplication in $\tilde{U}_{\text {res }}(V, W)$.

Proof. Due to the intertwining property with $\mathrm{d} \tilde{\Gamma}$ by definition $\tilde{\Gamma}$ is a representation of the operators of the form

$$
\left(\left(\begin{array}{ll}
a & 0 \\
c & d
\end{array}\right), z\right) \text { on } \check{S}(V \oplus \bar{W})
$$

and a representation of the operators of the form

$$
\left(\left(\begin{array}{ll}
a & b \\
0 & d
\end{array}\right), z\right), \quad \text { on } \check{S}(V \oplus \bar{W}) .
$$

With regard to the product decomposition in the definition of $\mathrm{d} \tilde{\Gamma}$, it remains to show that

$$
\tilde{\Gamma}\left(\left(\begin{array}{ll}
1 & 0 \\
c & 1
\end{array}\right), 1\right) \tilde{\Gamma}\left(\left(\begin{array}{ll}
1 & b \\
0 & 1
\end{array}\right), 1\right)=\tilde{\Gamma}\left(\left(\begin{array}{cc}
1 & b \\
c & 1+c b
\end{array}\right), \operatorname{det}^{-1}(1+c b)\right),
$$

if $\|c\|<1$ and $\|b\|<1$. To do this it suffices to show that

$$
\begin{aligned}
& \tilde{\Gamma}\left(\left(\begin{array}{ll}
1 & 0 \\
c & 1
\end{array}\right), 1\right) \tilde{\Gamma}\left(\left(\begin{array}{cc}
1 & t b \\
0 & 1
\end{array}\right), 1\right) \tilde{\Gamma}\left(\left(\begin{array}{rr}
1 & 0 \\
-c & 1
\end{array}\right), 1\right) \\
& =\tilde{\Gamma}\left(\left(\begin{array}{cc}
1-t b c & t b \\
-t c b c & 1+t c b
\end{array}\right), \operatorname{det}^{-1}(1+t c b)\right),
\end{aligned}
$$

if $\|c\|<1$ and $\|t b\|<1$. The left side is equal to

$$
\exp \left(\mathrm{d} \tilde{\Gamma}\left(\left(\begin{array}{cc}
-t b c & t b \\
-t c b c & t c b
\end{array}\right),-\operatorname{tr}(t c b)\right)\right)
$$

Each factor in the product decomposition of $\tilde{\Gamma}$ satisfies

$$
\frac{\mathrm{d}}{\mathrm{d} t} \tilde{\Gamma}(g(t))=\mathrm{d} \tilde{\Gamma}\left(\dot{g}(t) g^{-1}(t) \tilde{\Gamma}(g(t))\right.
$$


Combined with the intertwining property of $\mathrm{d} \tilde{\Gamma}$ and $\tilde{\Gamma}$ this implies

$$
\begin{aligned}
& \frac{\mathrm{d}}{\mathrm{d} t} \tilde{\Gamma}\left(\left(\begin{array}{cc}
1-t b c & t b \\
-t c b c & 1+t c b
\end{array}\right), \operatorname{det}^{-1}(1+t c b)\right) \\
& \quad=\mathrm{d} \tilde{\Gamma}\left(\left(\begin{array}{cc}
-b c & b \\
-c b c & c b
\end{array}\right),-\operatorname{tr}(c b)\right) \tilde{\Gamma}\left(\left(\begin{array}{cc}
1-t b c & t b \\
-t c b c & 1+t c b
\end{array}\right), \operatorname{det}^{-1}(1+t c b)\right) .
\end{aligned}
$$

As continuous operators from $\check{S}(V \oplus \bar{W})$ into $\check{S}(V \oplus \bar{W})$ for each $n$ and $m$, both sides are bounded operators from $S^{n}(V \oplus \bar{W})$ into $S^{m}(V \oplus \bar{W})$. Both sides are, moreover, holomorphic in $t$ and have the same derivatives at the point $t=0$, so they must be equal.

1.6. THEOREM. The representation $\mathrm{d} \tilde{\Gamma}$ of $\tilde{L}_{\mathrm{res}}(V, W)$ can be lifted to a unitary representation of $\tilde{U}_{\mathrm{res}}(V, W)$ on $S(V \oplus \bar{W})$.

Proof. Define $\theta: \tilde{L}_{\text {res }}(V, W) \rightarrow \tilde{L}_{\text {res }}(V, W)$,

$$
\left(\left(\begin{array}{ll}
a & b \\
c & d
\end{array}\right), z\right) \rightarrow-\left(\left(\begin{array}{rr}
1 & 0 \\
0 & -1
\end{array}\right)\left(\begin{array}{ll}
a^{*} & c^{*} \\
b^{*} & d^{*}
\end{array}\right)\left(\begin{array}{rr}
1 & 0 \\
0 & -1
\end{array}\right)^{-1}, \bar{z}\right)
$$

to be the involution of $\tilde{L}_{\text {res }}(V, W)$, whose fixpoints are elements of the Lie algebra of $\tilde{U}_{\text {res }}(V, W)$. The two meanings of $\mathrm{d} \tilde{\Gamma}$-on one side the image is an operator of $\hat{S}(V \oplus \bar{W})$ and on the other side an operator of $\check{S}(V \oplus \bar{W})$ and conversely-are related by

$$
\mathrm{d} \tilde{\Gamma}(\theta(-A))=(\mathrm{d} \Gamma(A))^{*}
$$

for all $A \in \tilde{L}_{\text {res }}(V, W)$. Let $\Theta$ be the corresponding group involution:

$$
\begin{aligned}
& \left(\left(\begin{array}{ll}
a & b \\
c & d
\end{array}\right), z\right) \\
& \rightarrow\left(\left(\begin{array}{rr}
1 & 0 \\
0 & -1
\end{array}\right)\left(\begin{array}{ll}
a^{*} & c^{*} \\
b^{*} & d^{*}
\end{array}\right)^{-1}\left(\begin{array}{rr}
1 & 0 \\
0 & -1
\end{array}\right)^{-1}, \frac{\operatorname{det}\left(1-d^{*-1} b^{*} a^{*-1} c^{*}\right)}{\bar{z}}\right) .
\end{aligned}
$$

Then

$$
\Theta\left(\left(\left(\begin{array}{ll}
a & b \\
c & d
\end{array}\right), z\right)^{-1}\right)
$$

gives

$$
\left(\left(\begin{array}{cc}
1 & -c^{*} d^{*-1} \\
0 & 1
\end{array}\right)\left(\begin{array}{cc}
a^{*}-c^{*} d^{*-1} b^{*} & 0 \\
0 & d^{*}
\end{array}\right)\left(\begin{array}{cc}
1 & 0 \\
-d^{*-1} b^{*} & 1
\end{array}\right), \bar{z}\right)
$$

So each factor in the product decomposition of $\tilde{\Gamma}$ obeys $\tilde{\Gamma}\left(\Theta\left(A^{-1}\right)\right)=(\tilde{\Gamma}(A))^{*}$, if on one side $\tilde{\Gamma}$ represents operators of the adjoint spaces of the other side. This implies

$$
\tilde{\Gamma}\left(A^{-1}\right)=(\tilde{\Gamma}(A))^{*}
$$

for all $A \in \tilde{U}_{\text {res }}(V, W)$. Combined with the preceding lemmas this guarantees that $\tilde{U}_{\text {res }}(V, W)$ is represented by isometric embeddings of $\check{S}(V \oplus \bar{W})$ into $S(V \oplus \bar{W})$. Such 
isometric operators extend uniquely to isometric operators of $S(V \oplus \bar{W})$. The preceding lemma shows the composition of two such continuous operators to be compatible with the group multiplication on the dense subspace $\breve{S}(V \oplus \bar{W})$ and, therefore, on the whole space $S(V \oplus \bar{W})$. This concludes the proof of the theorem.

The closure in $\hat{S}(V \oplus \bar{W})$ of any invariant subspace of $S(V \oplus \bar{W})$ is an invariant subspace of $\hat{S}(V \oplus \bar{W})$ with respect to the action of $\tilde{L}_{\text {res }}(V, W)$.

To determine the minimal weights, which occur in the $k$-fold tensor product of this representation, let us first define what we mean by minimal weight representation. Fix two orthonormal basis $\left\{v_{i}\right\}_{i \in \mathbb{N}}$ resp. $\left\{w_{i}\right\}_{i \in \mathbb{N}}$ of $V$ resp. $W$. All the definitions, we give now with respect to $V$ carry over to the corresponding situation for $W$. For all $n \in \mathbb{N}$ let $V_{n}$ be the subspace of $V$, which is spanned by the vectors $v_{1}, \ldots, v_{n}$. Let $b^{-}(V)$ be the subalgebra of $L(V)$, which maps $V_{n}$ into $V_{n}$ for all $n \in \mathbb{N} . b^{+}(V)$ is the adjoint subalgebra of $L(V) . n^{-}(V)$ is defined to be the subalgebra of $L(V)$, which maps $V_{1}$ into 0 and $V_{n+1}$ into $V_{n}$ for all $n \in \mathbb{N} . n^{+}(V)$ is the adjoint subalgebra. Let $\tilde{b}_{\text {res }}^{-}(V, W)$ be the subalgebra of $\tilde{L}_{\text {res }}(V, W)$, whose elements are of the form

$$
\left(\left(\begin{array}{ll}
a & 0 \\
c & d
\end{array}\right), z\right), \quad a \in b^{-}(V), d \in b^{+}(W), z \in \mathbb{C} .
$$

Let $\tilde{n}_{\text {res }}^{-}(V, W)$ be the subalgebra of $\tilde{L}_{\text {res }}(V, W)$, whose elements are of the form

$$
\left(\left(\begin{array}{ll}
a & 0 \\
c & d
\end{array}\right), 0\right), \quad a \in n^{-}(V), d \in n^{+}(W) .
$$

Let $\tilde{h}_{\text {res }}(V, W)$ be the maximal Abelian subalgebra of $\tilde{L}_{\text {res }}(V, W)$, whose elements are of the form

$$
\left(\left(\begin{array}{ll}
a & 0 \\
0 & d
\end{array}\right), z\right)
$$

with $a$ being diagonal with respect to $\left\{v_{i}\right\}_{i \in \mathbb{N}}$ and $d$ being diagonal with respect to $\left\{w_{i}\right\}_{i \in \mathbb{N}}$. A weight is an equivalence class of irreducible representations of $\tilde{h}_{\text {res }}(V, W)$, and a weight of a representation is the equivalence class of an irreducible subrepresentation of $\tilde{h}_{\text {res }}(V, W)$. All irreducible integrable unitary representations of $\tilde{h}_{\text {res }}(V, W)$ are classified by integers $\left\{m_{i}\right\}_{i \in \mathbb{N}},\left\{n_{1}\right\}_{i \in \mathbb{N}}$ and $n_{c}, m_{i}$ describes the action on $v_{i}, n_{i}$ describes the action on $w_{i}$ and $n_{c}$ is the central charge. Only a finite number of the $m_{i}$ 's and the $n_{i}$ 's are different from zero. Otherwise, the action would not be defined on all elements of $\tilde{h}_{\text {res }}(V, W)$. The subalgebra $\tilde{n}_{\text {res }}^{-}(V, W)$ of $\tilde{L}_{\text {res }}(V, W)$ induces an order on the unitary weights of $\tilde{h}_{\text {res }}(V, W)$ :

$$
\left(\left\{m_{i}\right\}_{i \in \mathbb{N}},\left\{n_{i}\right\}_{i \in \mathbb{N}}, n_{c}\right) \leqslant 0
$$

if and only if $m_{i} \geqslant m_{j}$ and $n_{i} \leqslant n_{j}$, whenever $i \leqslant j$.

A minimal weight vector of a representation, is a weight vector, which is annihilated by all elements of $\tilde{n}_{\text {res }}^{-}(V, W)$. 
To describe the $k$-fold tensor product of $\hat{S}(V \oplus \bar{W})$, we will use the following isomorphisms:

$$
\left.\hat{S}(V \oplus \bar{W}) \otimes \cdots \otimes \hat{S}(V \oplus \bar{W}) \simeq \hat{S}(V \oplus \bar{W}) \otimes \mathbb{C}^{k}\right) \simeq \hat{S}\left(V \otimes \mathbb{C}^{k} \oplus \bar{W} \otimes \overline{\mathbb{C}}^{k}\right)
$$

Set

$$
S_{k}^{n}:=S^{n}\left(V \otimes \mathbb{C}^{k} \oplus \bar{W} \otimes \overline{\mathbb{C}}^{k}\right) \quad \text { and } \quad S_{k}^{p, q}:=S^{p}\left(V \otimes \mathbb{C}^{k}\right) \otimes S^{q}\left(\bar{W} \otimes \mathbb{C}^{k}\right) .
$$

$S_{k}^{n}=\bigoplus_{p+q=n} S_{k}^{p, q}$ is a finite sum. So the Fréchet spaces $\hat{S}_{k}:=\Pi_{n} S_{k}^{n}$ and $\Pi_{p, q} S_{k}^{p, q}$ are canonically isomorphic.

There are canonical embeddings of Lie algebras:

$$
\begin{aligned}
& \mathrm{gl}(k, \mathbb{C}) \rightarrow \tilde{L}_{\mathrm{res}}\left(V \otimes \mathbb{C}^{k}, W \otimes \mathbb{C}^{k}\right) \text { and } \\
& \quad L(V), L(W) \rightarrow \tilde{L}_{\mathrm{res}}(V, W) \rightarrow \tilde{L}_{\mathrm{res}}\left(V \otimes \mathbb{C}^{k}, W \otimes \mathbb{C}^{k}\right)
\end{aligned}
$$

We identify image and preimage of this embeddings and of the corresponding group embeddings. Let $e_{1}, \ldots, e_{k}$ be the canonical orthonormal basis of $\mathbb{C}^{k}$. Set

$$
\begin{aligned}
& \Delta_{\mu}(v):=\operatorname{det}\left[\begin{array}{ccc}
v_{1} \otimes e_{1} & \cdots & v_{1} \otimes e_{\mu} \\
\vdots & & \vdots \\
v_{\mu} \otimes e_{1} & \cdots & v_{\mu} \otimes e_{\mu}
\end{array}\right], \quad 0 \leqslant \mu \leqslant k, \\
& \Delta_{v}(\bar{w}):=\operatorname{det}\left[\begin{array}{ccc}
\bar{w}_{1} \otimes \bar{e}_{k-v+1} & \cdots & \bar{w}_{1} \otimes \bar{e}_{k} \\
\vdots & & \vdots \\
\bar{w}_{v} \otimes \bar{e}_{k-v+1} & \cdots & \bar{w}_{v} \otimes \bar{e}_{k}
\end{array}\right], \quad 0 \leqslant v \leqslant k,
\end{aligned}
$$

such that both $\Delta_{\mu}(v)$ and $\Delta_{v}(\bar{w})$ are in $S_{k}$. Now

$$
\Delta_{1}^{\alpha_{1}}(v) \cdots \Delta_{\mu}^{\alpha_{\mu}}(v) \Delta_{1}^{\beta_{1}}(\bar{w}) \cdots \Delta_{v}^{\beta_{v}}(\bar{w}), \mu+v \leqslant k, \alpha_{\mu}, \beta_{v} \in \mathbb{N}_{0}
$$

are minimal weight vectors of $\hat{S}_{k}$. The corresponding weight is

$$
m_{i}=\sum_{\mu \geqslant i} \alpha_{\mu}, \quad n_{i}=-\sum_{\nu \geqslant i} \beta_{v} \quad \text { and } \quad n_{c}=k
$$

This leads to the following lemma.

1.7. LEMMA. For all weights $\left(\left\{m_{i}\right\}_{i \in \mathbb{N}},\left\{n_{i}\right\}_{i \in \mathbb{N}}, n_{c}\right) \leqslant 0$, with

$$
n_{c} \geqslant \min \left\{i \mid m_{j}=0 \text { for all } j>i\right\}+\min \left\{i \mid n_{j}=0 \text { for all } j>i\right\} \text {, }
$$

there is a minimal weight vector in $\hat{S}_{n_{c}}$.

Kashiwara and Vergne [7] showed, that if $V$ and $W$ are finite dimensional, these are all minimal weight vectors and the representation of $\mathrm{U}(V, W)$ is completely reducible. We want to extend these results to the infinite dimensional case.

Now let $\Sigma$ be the set of all equivalence classes of irreducible unitary representations of $\mathrm{U}(k, \mathbb{C})$, and for all $\lambda \in \Sigma$ let $V_{\lambda}$ be a representative. With respect to the maximal toral subalgebra of $\operatorname{gl}(k, \mathbb{C})$ of all diagonal matrices, the weights of $\operatorname{gl}(k, \mathbb{C})$ can be described 
by $k$ numbers $n_{1}, \ldots, n_{k}$. The Borel subalgebra $b^{-}$of all upper-triangular matrices induces an order on the weight space: $\left(n_{1}, \ldots, n_{k}\right) \leqslant 0$ if and only if $n_{1} \geqslant n_{2} \geqslant \cdots \geqslant n_{k}$. The equivalence classes of irreducible unitary representations of $\mathrm{U}(k, \mathbb{C})$ are isomorphic to the equivalence classes of all unitary lowest weight representations, and these classes are isomorphic to all integral weights, which are smaller than zero. So $\Sigma$ can be identified with this set.

For a unitary representation $H$ of $\mathrm{U}(k, \mathbb{C})$ let $L_{\lambda}(H)$ be the Hilbert space of $\operatorname{HOM}_{\mathrm{U}(k, \mathrm{C})}\left(V_{\lambda}, H\right)$ with the Hermitian form $\langle a, b\rangle:=\operatorname{tr}\left(a^{*} b\right)$. In case the vector space $H$ is only a locally convex vector space, let $L_{\lambda}(H)$ be the corresponding locally convex vector space.

Let $\hat{H}_{k}$ be the kernel of all operators

$$
\mathrm{d} \tilde{\Gamma}\left(\left(\begin{array}{ll}
0 & 0 \\
c & 0
\end{array}\right), 0\right)
$$

with $c: V \rightarrow W$ an arbitrary Hilbert-Schmidt operator. Set $c_{i, j}: V \rightarrow W$ for the finite rank operator, which maps $v_{j}$ onto $w_{i}$ and is zero otherwise. Set

$$
H_{k}=\hat{H}_{k} \cap S_{k} \quad \text { and } \quad H_{k}^{p, q}=\hat{H}_{k} \cap S_{k}^{p, q} \text {. }
$$

The subspace $\check{H}_{k}:=\hat{H}_{k} \cap \breve{S}_{k}$ is dense in $\hat{H}_{k}$, because $\hat{H}_{k}$ is an invariant closed subspace with respect to

$$
P:=\mathrm{d} \tilde{\Gamma}\left(\left(\begin{array}{ll}
1 & 0 \\
0 & 0
\end{array}\right), 0\right)
$$

and

$$
Q:=\mathrm{d} \tilde{\Gamma}\left(\left(\begin{array}{rr}
0 & 0 \\
0 & -1
\end{array}\right), 0\right)
$$

$\mathrm{U}(k, \mathbb{C})$ and $\tilde{\mathrm{U}}_{\text {res }}(V, W)$ commute as subgroups of $\tilde{\mathrm{U}}_{\text {res }}\left(V \otimes \mathbb{C}^{k}, W \otimes \mathbb{C}^{k}\right)$. Hence $L_{\lambda}\left(S_{k}\right)$ is a unitary representation of $\tilde{\mathrm{U}}_{\text {res }}(V, W)$ and $L_{\lambda}\left(\hat{S}_{k}\right)$ is a representation of $\tilde{L}_{\text {res }}(V, W)$. Moreover, $L_{\lambda}\left(H_{k}\right)$ is a unitary representation of $\mathrm{U}(V) \times \mathrm{U}(W)$.

Let $e_{\mu, v}, 1 \leqslant \mu, v \leqslant k$ be the canonical basis of $\mathrm{gl}(k, \mathbb{C})$. Set

$$
P_{\mu, v}:=\sum_{i} a^{*}\left(v_{i} \otimes e_{\mu}\right) a\left(v_{i} \otimes e_{v}\right)=\mathrm{d} \tilde{\Gamma}\left(\left(\begin{array}{cc}
1 \otimes e_{\mu, v} & 0 \\
0 & 0
\end{array}\right), 0\right)
$$

and

$$
Q_{\mu, \nu}:=-\sum_{j} a^{*}\left(\bar{w}_{j} \otimes \bar{e}_{v}\right) a\left(\bar{w}_{j} \otimes \bar{e}_{\mu}\right)=\mathrm{d} \tilde{\Gamma}\left(\left(\begin{array}{cc}
0 & 0 \\
0 & 1 \otimes e_{\mu, v}
\end{array}\right), 0\right)
$$

for all $1 \leqslant \mu, v \leqslant k$. From the definition of $\mathrm{d} \tilde{\Gamma}$ it follows, that the $P_{\mu, \nu}$ and $Q_{\mu, \nu}$ satisfy the relations

$$
\mathrm{d} \tilde{\Gamma}\left(e_{\mu, v}\right)=P_{\mu, v}+Q_{\mu, \nu}, \quad P_{\mu, \nu}^{*}=P_{\mu, v} \quad \text { and } \quad Q_{\mu, \nu}^{*}=Q_{v, \mu} .
$$


1.8. LEMMA. For all $\lambda \in \Sigma$ set $p(\lambda):=\Sigma_{n_{\mu} \geqslant 0} n_{\mu}$ and $q(\lambda):=-\Sigma_{n_{v} \leqslant 0} n_{v}$. Then $L_{\lambda}\left(H_{k}^{p, q}\right)=0$ if $p \neq p(\lambda)$ or $q \neq q(\lambda) . L_{\lambda}\left(H_{k}^{p(\lambda), q(\lambda)}\right)$ is an irreducible representation of $C(V) \times C(W)$.

Proof.

$$
\begin{aligned}
\sum_{i, j} & a^{*}\left(v_{j} \otimes e_{\mu}\right) a^{*}\left(\bar{w}_{i} \otimes \bar{e}_{\mu}\right) \mathrm{d} \tilde{\Gamma}\left(\left(\begin{array}{cc}
0 & 0 \\
c_{i, j} & 0
\end{array}\right), 0\right) \\
& =-\sum_{i, j, v} a^{*}\left(v_{j} \otimes e_{\mu}\right) a\left(v_{j} \otimes e_{v}\right) a^{*}\left(\bar{w}_{i} \otimes \bar{e}_{\mu}\right) a\left(\bar{w}_{i} \otimes \bar{e}_{v}\right) \\
& =\sum_{v=1}^{k} P_{\mu, \nu} Q_{v, \mu} .
\end{aligned}
$$

From the definition it follows, that $H_{k}^{p, q}$ must be a subset of the kernel of the operators $\sum_{v=1}^{k} P_{\mu, v} Q_{v, \mu}$ for all $1 \leqslant \mu \leqslant k$. Now let $v$ be a minimal weight vector with respect to the action of $\operatorname{gl}(k, \mathbb{C})$. Then $\left(P_{v, \mu}+Q_{v, \mu}\right) v=0$ for all $1 \leqslant v<\mu \leqslant k$. It follows that $-P_{\mu, v}^{*} v=Q_{v, \mu} v$. If $v$ is an element of $H_{k}^{p, q}$ it therefore has to obey

$$
\left(-P_{1,1} Q_{1,1}+\sum_{v=2}^{k} P_{1, v} P_{1, v}^{*}\right) v=0 .
$$

Now $P_{1,1}$ is a positive operator and $Q_{1,1}$ a negative operator. So $P_{1,1} Q_{1,1} v$ must be zero. Now it follows, that for all $v \in V, w \in W a\left(v \otimes e_{1}\right) a\left(\bar{w} \otimes \bar{e}_{1}\right) v=0$. Using this methods for the other operators $P_{\mu, v}$ iteratively $(\mu=1, \ldots, k)$ one finally gets $P_{\mu, \mu} Q_{\mu, \mu} v=0$ for all $\mu=1, \ldots, k$. Now $\left(P_{\mu, \mu}+Q_{\mu, \mu}\right) v$ must be equal to $n_{\mu} v$. On the other hand, the $P_{\mu, \mu}$ are positive operators and the $Q_{\mu, \mu}$ are negative operators. This gives

$$
\begin{array}{lrl}
P_{\mu, \mu} v=n_{\mu} v, & Q_{\mu, \mu} v=0, & \text { if } n_{\mu} \geqslant 0, \\
P_{\mu, \mu} v=0, & Q_{\mu, \mu} v=n_{\mu} v, & \text { if } n_{\mu} \leqslant 0 .
\end{array}
$$

This implies

$$
\sum_{\mu=1}^{k} P_{\mu, \mu} v=p(\lambda) v \text { and }-\sum_{\mu=1}^{k} Q_{\mu, \mu} v=q(\lambda)
$$

which is equivalent to $v \in H_{k}^{p(\lambda), q(\lambda)}$ and the first part of the lemma is proved.

Let $\mathbb{C}_{+}^{k}$ and $\mathbb{C}_{-}^{k}$ be the span of all $e_{\mu}$, such that $n_{\mu}>0$ and $n_{\mu}<0$, respectively. To prove the second part, it suffices to show that the subspace of $H_{k}^{p(\lambda), q(\lambda)}$ of all minimal weight vectors with weight $\lambda$ is irreducible with respect to the action of $C(V) \times C(W)$. The proof of the first part of the lemma shows, that this space is a subspace of $S^{p(\lambda)}\left(V \otimes \mathbb{C}_{+}^{k}\right) \otimes S^{q(\lambda)}\left(\bar{W} \otimes \overline{\mathbb{C}}_{-}^{k}\right)$, which is, of course, an irreducible representation of $C(V) \times C(W) \times \operatorname{gl}\left(\mathbb{C}_{+}^{k}\right) \times \mathrm{gl}\left(\mathbb{C}_{-}^{k}\right)$. Thus, the minimal weight vectors are irreducible with respect to $C(V) \times C(W)$. This concludes the proof of the lemma.

1.9. PROPOSITION. $L_{\lambda}\left(\hat{S}_{k}\right)$ is an irreducible representation of $\tilde{L}_{\mathrm{res}}(V, W) . L_{\lambda}\left(S_{k}\right)$ is an irreducible unitary representation of $\tilde{U}_{\mathrm{res}}(V, W)$. 
Proof. Set

$$
P:=\mathrm{d} \tilde{\Gamma}\left(\left(\begin{array}{ll}
1 & 0 \\
0 & 0
\end{array}\right), 0\right) \text { and } Q:=\mathrm{d} \Gamma\left(\left(\begin{array}{rr}
0 & 0 \\
0 & -1
\end{array}\right), 0\right) .
$$

Each closed subspace of $\hat{S}_{k}$, which is invariant with respect to $P$ and $Q$ contains its orthogonal components in $S_{k}^{p, q}$. So every invariant subspace of $L_{\lambda}\left(\hat{S}_{k}\right)$ meets $L_{\lambda}\left(\hat{H}_{k}\right)=L_{\lambda}\left(H_{k}^{p(\lambda), q(\lambda)}\right)$, because all operators of the form

$$
\mathrm{d} \tilde{\Gamma}\left(\left(\begin{array}{ll}
0 & 0 \\
c & 0
\end{array}\right), 0\right)
$$

reduce $p$ and $q$ by one. Due to the preceeding lemma it then must contain this space. By the unitarity of the representation of $\tilde{U}_{\text {res }}(V, W)$ on $L_{\lambda}\left(S_{k}\right)$ now it follows, that $L_{\lambda}\left(\hat{S}_{k}\right)$ is an irreducible representation of $\tilde{L}_{\mathrm{res}}(V, W)$.

1.10. THEOREM. $S_{k}$ is completely reducible into unitary lowest weight representations of $\tilde{\mathrm{U}}_{\mathrm{res}}(V, W)$. The decomposition is explicitly given by $S_{k}=\bigoplus_{\lambda \in \Sigma}\left(\bar{V}_{\lambda} \otimes L_{\lambda}\left(S_{k}\right)\right.$ (completion of the infinite sum). The $L_{\lambda}\left(S_{k}\right)$ are all distinct.

Proof. It remains to show, that the $L_{\lambda}\left(S_{k}\right)$ are lowest weight representations and are all distinct. Now let

$$
\begin{array}{lll}
p_{i}=n_{i}, & q_{i}=0, & \text { if } n_{i} \geqslant 0, \\
p_{i}=0, & q_{i}=-n_{i}, & \text { if } n_{i} \leqslant 0 .
\end{array}
$$

Now $\Delta_{1}^{p_{1}-p_{2}}(v) \Delta_{2}^{p_{2}-p_{3}}(v) \cdots \Delta_{i}^{p_{k}}(v) \Delta_{1}^{q_{k}-q_{k-1}}(\bar{w}) \Delta_{2}^{q_{k}-1}-q_{k-2}(\bar{w}) \cdots \Delta_{k}^{q_{1}}(\bar{w})$ is a minimal weight vector with respect to the action of both $\tilde{L}_{\mathrm{res}}(V, W)$ and $\mathrm{gl}(k, \mathbb{C})$. The map

$$
\lambda \rightarrow\left(\left(p_{1}, \ldots, p_{k}, 0, \ldots\right),\left(-q_{k}, \ldots,-q_{1}, 0, \ldots\right), k\right)
$$

from $\Sigma$ into the weights of $\tilde{L}_{\mathrm{res}}(V, W)$ is injective. So all $L_{\lambda}\left(S_{k}\right)$ are distinct.

1.11. Remark. In $[8]$ and in $[9,10]$ it was shown that all unitary lowest weight representations of $\mathrm{U}(V, W)$ are contained in $S_{k}$ for some $k$, if $V$ and $W$ are finite dimensional. If $V$ or $W$ is not finite dimensional in all weights $\left(\left\{m_{i}\right\}_{i \in \mathbb{N}},\left\{n_{i}\right\}_{i \in \mathbb{N}}, n_{c}\right)$, only a finite number of the $m_{i}^{\prime}$ s and the $n_{i}^{\prime} \mathrm{s}$ are different from zero. The embeddings of finite dimensional groups $\mathrm{U}\left(V^{\prime}, W^{\prime}\right)$ into $\tilde{\mathrm{U}}_{\text {res }}(V, W)$ then guarantee that all unitary lowest weight representations of $\tilde{U}_{\text {res }}(V, W)$ are contained in some $S_{k}$.

\section{The Representations of $\operatorname{Sp}(V)$}

In this part we determine the decomposition of the tensor products of the 'SegalShale-Weil representation' of the metaplectic group. Let us first recall the definition of the symplectic group of some Hilbert space $V$. This definition is due to Shale [3], who found the corresponding representation, but our point of view is more inspired by the work of G. Segal [1].

Let $V$ be a complex Hilbert space and $V_{\mathbb{R}}$ the corresponding real Hilbert space. Then the complexification of $V_{\mathbb{R}}$ is canonically isomorphic to $V \oplus \bar{V}$. Hence, all real 
linear operators of $V_{\mathbb{R}}$ can be thought of as complex linear operators of $V \oplus \bar{V}$, which commute with the complex conjugation induced by $V_{\mathbb{B}}$. Let $\lambda: V \rightarrow \bar{V}$ and $\lambda: \bar{V} \rightarrow V$ be the canonical antilinear isomorphisms. Then complex conjugation is expressed as

$$
\Lambda=\left(\begin{array}{cc}
0 & \lambda^{*} \\
\lambda & 0
\end{array}\right)
$$

The symplectic group is defined to be the subgroup of $G L\left(V_{\mathbb{R}}\right)$, which leaves the antisymmetric nondegenerated form induced by the multiplication with $i$ in $V$ invariant. The operator of multiplication with $i$ in $V$ induces of course the operator

$$
\left(\begin{array}{cc}
i & 0 \\
0 & -i
\end{array}\right) \text { on } V \oplus \bar{V}
$$

Hence $\operatorname{Sp}(V)$ is the commutant of $\Lambda$ in $\mathrm{U}(V, \bar{V})$.

Set $\mathrm{Sp}_{\text {res }}(V):=\operatorname{Sp}(V) \cap U_{\text {res }}(V, \bar{V})$. In the definition of the cocycle $c\left(A_{1}, A_{2}\right)$ of the central extension of $U_{\text {res }}(V, \bar{V})$ it was shown that $d_{1}^{-1} d_{3} d_{2}^{-1}$ is of the form $1+d_{1}^{-1} c_{1} b_{2} d_{2}^{-1} \cdot d_{1}^{-1} c_{1} b_{2} d_{2}^{-1}$ is a trace class operator with norm smaller than 1 . Hence the cocycle $c^{\prime}\left(A_{1}, A_{2}\right):=\operatorname{det}^{-1 / 2}\left(d_{1}^{-1} d_{3} d_{2}^{-1}\right)$ is well defined. Let

$$
\tilde{\mathrm{Sp}}_{\text {res }}(V):=\left\{\left(\left(\begin{array}{cc}
a & b \\
\bar{b} & \bar{a}
\end{array}\right), z\right), \quad \text { with } z \bar{z}=\operatorname{det}^{1 / 2}\left(1-a^{t^{-1}} \overline{b^{t}} b \bar{a}^{-1}\right)\right\}
$$

be the central extension of $\operatorname{Sp}_{\mathrm{res}}(V)$ induced by this cocycle.

2.1. Remark. If $V$ is finite dimensional this central extension is trivial over a double covering group of $\mathrm{Sp}(V)$, the metaplectic group of $V$ (see e.g. Segal [1] and Kashiwara and Vergne [7]).

Set $\tilde{\Lambda}_{\text {res }}(V)$ for the corresponding subalgebra of $\tilde{L}_{\text {res }}(V, \bar{V})$. It consists of all elements

$$
\left(\left(\begin{array}{ll}
a & b \\
c & d
\end{array}\right), z\right)
$$

which satisfy

$$
\left(\begin{array}{cc}
0 & -\lambda^{*} \\
\lambda & 0
\end{array}\right)\left(\begin{array}{ll}
a & b \\
c & d
\end{array}\right)^{*}\left(\begin{array}{cc}
0 & -\lambda^{*} \\
\lambda & 0
\end{array}\right)^{-1}=-\left(\begin{array}{ll}
a & b \\
c & d
\end{array}\right) .
$$

This is equivalent to $b=b^{t}, c=c^{t}, d=-a^{t}$. The following map

$$
\begin{aligned}
\mathrm{d} \tilde{\Gamma} & \left(\left(\begin{array}{cc}
a & b \\
c & -a^{t}
\end{array}\right), z\right) \\
:= & \sum_{i, j} a^{*}\left(v_{i}\right) a\left(v_{j}\right)\left\langle v_{i}, a v_{j}\right\rangle+ \\
& +\frac{1}{2} \sum_{i, j} a^{*}\left(v_{i}\right) a^{*}\left(v_{j}\right)\left\langle v_{j}, b \bar{v}_{i}\right\rangle- \\
& \quad-\frac{1}{2} \sum_{i, j} a\left(v_{i}\right) a\left(v_{j}\right)\left\langle\bar{v}_{j}, c v_{i}\right\rangle+\frac{z}{2} 1
\end{aligned}
$$


from $\tilde{\Lambda}_{\text {res }}(V)$ into the continuous operators of $\hat{S}(V)$ defines a representation of $\tilde{\Lambda}_{\text {res }}(V)$. This sum converges only pointwise. Because of the linearity of $a^{*}(v)$ and the antilinearity of $a(v)$ in $v, \mathrm{~d} \tilde{\Gamma}$ does not depend on the choice of the orthonormal basis $\left\{v_{i}\right\}_{i \in \mathbb{N}}$. The arguments for $\mathrm{d} \tilde{\Gamma}$ being a representation of $\tilde{L}_{\text {res }}(V, W)$ carry over to the Lie algebra $\tilde{\Lambda}_{\text {res }}(V)$ showing again $d \tilde{\Gamma}$ to be a representation.

All bounded operators

$$
\left(\begin{array}{ll}
a & b \\
c & d
\end{array}\right) \text { of } V \oplus \bar{V}
$$

which satisfy

$$
\left(\begin{array}{cc}
0 & -\lambda^{*} \\
\lambda & 0
\end{array}\right)\left(\begin{array}{cc}
a & b \\
c & d
\end{array}\right)^{*}\left(\begin{array}{cc}
0 & -\lambda^{*} \\
\lambda & 0
\end{array}\right)^{-1}=\left(\begin{array}{ll}
a & b \\
c & d
\end{array}\right)^{-1}
$$

are elements of the complexification of $\operatorname{Sp}(V)$. If $d$ is invertible there exists a product decomposition

$$
\left(\begin{array}{cc}
1 & b d^{-1} \\
0 & 1
\end{array}\right)\left(\begin{array}{cc}
a-b d^{-1} c & 0 \\
0 & d
\end{array}\right)\left(\begin{array}{cc}
1 & 0 \\
d^{-1} c & 1
\end{array}\right)
$$

inside the complexification of $\mathrm{Sp}(V)$. Indeed by straightforward calculation one gets that $b d^{-1}$ and $d^{-1} c$ are symmetric and that $a-b d^{-1} c$ is equal to $d^{-2}$. If $d$ is invertible this product decomposition, hence determines completely the representation map on the complexification of $\tilde{\mathrm{S}} \mathrm{p}_{\text {res }}(V)$

$$
\begin{aligned}
& \tilde{\Gamma}\left(\left(\begin{array}{ll}
a & b \\
c & d
\end{array}\right), z\right) \\
& \quad:=\exp \left(\mathrm{d} \tilde{\Gamma}\left(\left(\begin{array}{cc}
0 & b d^{-1} \\
0 & 0
\end{array}\right), 0\right)\right) \tilde{\Gamma}\left(\left(\begin{array}{cc}
a-b d^{-1} c & 0 \\
0 & d
\end{array}\right), z\right) \exp \left(\mathrm{d} \tilde{\Gamma}\left(\left(\begin{array}{cc}
0 & 0 \\
d^{-1} c & 0
\end{array}\right), 0\right)\right) .
\end{aligned}
$$

The middle side is defined to be the canonical representation of $d^{t^{-1}}$ (which is equal to $a-b d^{-1} c$ ) times $z$. The intertwining properties of $\tilde{\Gamma}$ and $\mathrm{d} \tilde{\Gamma}$ are the same as in the chapter before.

\subsection{LEMMA}

$$
\exp \left(\mathrm{d} \tilde{\Gamma}\left(\left(\begin{array}{ll}
0 & b \\
0 & 0
\end{array}\right), 0\right)\right)
$$

is a continuous operator from $\breve{S}(V)$ into $S(V)$ if

$$
\|b\|<1 . \exp \left(\mathrm{d} \tilde{\Gamma}\left(\left(\begin{array}{ll}
0 & 0 \\
c & 0
\end{array}\right), 0\right)\right)
$$

is a continuous operator from $S(V)$ into $\hat{S(V)}$, if $\|c\|<1$. 
The proof is analogous to the proof of Lemma 1.4 after changing $p_{n}\left(b^{*} b\right)$ into $\frac{1}{2} \operatorname{tr}\left(\left(b^{*} b\right)^{n}\right)$ and $\beta$ into $\left.1 / 2\|b\|^{2}\right) \operatorname{tr}\left(b^{*} b\right)$. For all $x \in S^{p}(V)$ this yields the estimate

$$
\langle\exp (b) x, \exp (b) x\rangle \leqslant\left(\begin{array}{c}
p+\beta \\
p
\end{array}\right)\left(\frac{1}{1-\|b\|}\right)^{p+\beta}\langle x, x\rangle .
$$

The preceding lemma shows again that the image of $\tilde{\Gamma}$ consists either of continuous operators from $\breve{S}(V)$ into $S(V)$ or of continuous operators from $S(V)$ into $\hat{S}(V)$ or, of course, of continuous operators from $\hat{S}(V \oplus \bar{W})$ into $\hat{S}(V \oplus \bar{W})$. The actual intended meaning should be clear from the context.

2.3. LEMMA. The composition of two such operators is compatible with the group multiplication in $\widetilde{\mathrm{p}}_{\mathrm{res}}(V)$.

This lemma and the following theorem are given without proof, because the arguments are analogous of those of Lemma 1.5 and Theorem 1.6.

2.4. THEOREM. The representation $\mathrm{d} \tilde{\Gamma}$ of $\tilde{\Lambda}_{\mathrm{res}}(V)$ can be lifted to a unitary representation of $\tilde{\mathrm{S}}_{\mathrm{pres}}(V)$ on $\mathrm{S}(V)$.

\subsection{Remark. Consider}

$$
U=\frac{1}{\sqrt{2}}\left(\begin{array}{cc}
1 & 1 \\
i & -i
\end{array}\right)
$$

as an element of $\mathrm{U}(V \oplus V)$. Let $U$ also denote the canonical representation of $U$ on $\hat{S}(V \oplus V)$. The intertwining relations

$$
\begin{aligned}
& U a^{*}(v) U^{-1}=a^{*}(U v), \quad v \in V, \\
& U a(v) U^{-1}=a\left(U^{*-1} v\right), \quad v \in V
\end{aligned}
$$

lead to the following relation between the representation $\mathrm{d} \tilde{\Gamma}_{\tilde{L}_{\text {res }}(V, \bar{V})}$ of the subalgebra $\tilde{\Lambda}_{\text {res }}(V)$ of $\tilde{L}_{\text {res }}(V, \bar{V})$ on $\hat{S}(V \oplus V)$ and the 2-fold tensor product $\mathrm{d} \tilde{\Gamma}_{\tilde{\Lambda}_{\text {res }}(V)}^{2}$ of the

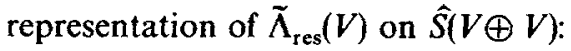

$$
U \mathrm{~d} \tilde{\Gamma}_{\tilde{L}_{\text {res }}(V, \bar{V})} U^{-1}=\mathrm{d} \tilde{\Gamma}_{\tilde{\Lambda}_{\text {res }}(V)}^{2} .
$$

So these representations are unitarily equivalent.

To determine the minimal weights which occur in the $k$-fold tensor product of this representation, let us first define what we mean by minimal weight representation of $\tilde{\mathrm{S}} \mathrm{p}_{\text {res }}(V)$. Fix an orthonormal basis $\left\{v_{i}\right\}_{i \in \mathbb{N}}$ of $V$. Let $\tilde{n}_{\text {res }}^{-}(V)$ be the subalgebra of $\tilde{\Lambda}_{\text {res }}(V)$, whose elements are of the form

$$
\left(\left(\begin{array}{cc}
a & 0 \\
c & -a^{t}
\end{array}\right), 0\right), \quad a \in n^{-}(V)
$$


Let $\tilde{h}_{\text {res }}(V)$ be the maximal Abelian subalgebra of $\tilde{\Lambda}_{\text {res }}(V)$, whose elements are of the form

$$
\left(\left(\begin{array}{cc}
a & 0 \\
0 & -a^{t}
\end{array}\right), z\right)
$$

with $a$ diagonal with respect to $\left\{v_{i}\right\}_{i \in \mathbb{N}}$. A weight is an equivalence class of irreducible representations of $\tilde{h}_{\text {res }}(V)$, and a weight of a representation is the equivalence class of an irreducible subrepresentation of $\tilde{h}_{\text {res }}(V)$. All irreducible integrable unitary representations of $\tilde{h}_{\text {res }}(V)$ are classified by integers $\left\{m_{i}\right\}_{i \in \mathbb{N}}$ and $n_{c} . m_{i}$ describes the action on $v_{i}$ and $n_{c} / 2$ is the central charge. Only a finite number of the $m_{i}$ 's are different from zero. Otherwise, the action would again not be defined on all elements of $\tilde{h}_{\text {res }}(V)$. The subalgebra $\tilde{n}_{\text {res }}^{-}(V)$ of $\tilde{\Lambda}_{\text {res }}(V)$ induce an order on the unitary weights of $\tilde{h}_{\text {res }}(V)$ :

$$
\left(\left\{m_{i}\right\}_{i \in \mathbb{N}}, n_{c}\right) \leqslant 0 \text { if and only if } m_{i} \geqslant m_{j} \text { whenever } i \leqslant j .
$$

A minimal weight vector of a representation is a weight vector which is annihilated by all elements of $\tilde{n}_{\text {res }}^{-}(V)$.

To describe the $k$-fold tensor product of $\hat{S}(V)$ we will use the following isomorphisms

$$
\hat{S}(V) \otimes \cdots \otimes \hat{S}(V) \simeq \hat{S}\left(V \otimes \mathbb{C}^{k}\right)
$$

Set $S_{k}^{n}:=S^{n}\left(V \otimes \mathbb{C}^{k}\right)$.

The complexification of the Lie algebra of $\mathrm{O}(k, \mathbb{R})$ is $\operatorname{so}(k, \mathbb{C})$. There are canonical embeddings of Lie algebras:

$$
\operatorname{so}(k, \mathbb{C}) \rightarrow \tilde{\Lambda}_{\text {res }}\left(V \otimes \mathbb{C}^{k}\right) \text { and } \quad L(V) \rightarrow \tilde{\Lambda}_{\text {res }}(V) \rightarrow \tilde{\Lambda}_{\text {res }}\left(V \otimes \mathbb{C}^{k}\right) .
$$

We identify image and preimage of this embeddings and of the corresponding group embeddings.

Now let $\Sigma$ be the set of all equivalence classes of irreducible unitary representations of $O(k, \mathbb{R})$ and for all $\lambda \in \Sigma$ let again $V_{\lambda}$ denote a representative. For a unitary representation $H$ of $\mathrm{O}(k, \mathbb{R})$ let $L_{\lambda}(H)$ be the Hilbert space of $\operatorname{HOM}_{\mathrm{O}(k, \mathbb{R})}\left(V_{\lambda}, H\right)$ with the Hermitian form $\langle a, b\rangle:=\operatorname{tr}\left(a^{*} b\right)$. In case the vector space $H$ is only a locally convex vector space, let $L_{\lambda}(H)$ be the corresponding locally convex vector space.

Let $\hat{H}_{k}$ be the kernel of all operators

$$
\mathrm{d} \tilde{\Gamma}\left(\left(\begin{array}{ll}
0 & 0 \\
c & 0
\end{array}\right), 0\right)
$$

with $c: V \rightarrow \bar{V}$ an arbitrary Hilbert-Schmidt operator. Let $c_{i, j}$ be the sum of the finite rank operators from $V$ to $\bar{V}$, which maps $v_{j}$ onto $\bar{v}_{i}$ and $v_{i}$ onto $\bar{v}_{j}$, respectively and which are zero otherwise. Set $H_{k}=\hat{H}_{k} \cap S_{k}$ and $H_{k}^{n}=\hat{H}_{k} \cap S_{k}^{n}$. The subspace $\check{H}_{k}:=\hat{H}_{k} \cap \check{S}_{k}$ is dense in $\hat{H}_{k}$, because $\hat{H}_{k}$ is an invariant closed subspace of $\hat{S}_{k}$ with respect to the action of

$$
N:=\mathrm{d} \tilde{\Gamma}\left(\left(\begin{array}{rr}
1 & 0 \\
0 & -1
\end{array}\right), 0\right) .
$$


The subgroups $\mathrm{O}(k, \mathbb{R})$ and $\widetilde{\mathrm{Sp}}_{\mathrm{pes}}(V)$ of $\widetilde{\mathrm{Sp}}_{\mathrm{pes}}\left(v \otimes \mathbb{C}^{k}\right)$ commute. Hence, $L_{\lambda}\left(\hat{S}_{k}\right)$ is a representation of $\tilde{\Lambda}_{\text {res }}(V)$ and $L_{\lambda}\left(S_{k}\right)$ a unitary representation of $\tilde{S}_{\text {res }}(V)$. Moreover, $L_{\lambda}\left(\hat{H}_{k}\right)$ is a representation of $L(V)$ and $L_{\lambda}\left(H_{k}\right)$ a unitary representation of $U(V)$.

In order to investigate these representations, we follow the discussion of Kashiwara and Vergne [7]. In the case $k=2 l+1, \mathrm{O}(k, \mathbb{R})$ is the direct product of $\operatorname{SO}(k, \mathbb{R})$ and $Z_{2}$ and an element of $\Sigma$ is determined by two irreducible representations of these groups. We will use the decomposition $\mathbb{C}^{k}=\mathbb{C}^{l} \oplus \mathbb{C}^{l} \oplus \mathbb{C}$ in order to express the elements of $\operatorname{gl}(k, \mathbb{C})$ and $\operatorname{so}(k, \mathbb{C})$.

$$
\text { Set } J:=\left(\begin{array}{ccc}
0 & 1 & 0 \\
1 & 0 & 0 \\
0 & 0 & 1
\end{array}\right)
$$

and

$$
\begin{aligned}
& \mathrm{O}(k, \mathbb{C}):=\left\{g \in \mathrm{GL}(k, \mathbb{C}) \mid g^{t} J g=J\right\}, \\
& \mathrm{SO}(k, \mathbb{C}):=\{g \in \mathrm{O}(k, \mathbb{C}) \mid \operatorname{det}(g)=1\}, \\
& \operatorname{so}(k, \mathbb{C}):=\left\{x \in \operatorname{sl}(k, \mathbb{C}) \mid x^{t} J+J x=0\right\} .
\end{aligned}
$$

By definition the elements of $\operatorname{so}(k, \mathbb{C})$ are of the form

$$
\left(\begin{array}{ccc}
a & b & e \\
c & -a^{t} & f \\
-f^{t} & -e^{t} & 0
\end{array}\right)
$$

with $b$ and $c$ being skew symmetric. We consider the Borel subalgebra

$$
b_{s o}^{-}:=\left\{\left(\begin{array}{ccc}
a & b & e \\
0 & -a^{t} & 0 \\
0 & -e^{t} & 0
\end{array}\right), a \text { upper triangular }\right\}
$$

and the Cartan subalgebra

$$
h_{s o}:=\left\{\left(\begin{array}{ccc}
a & 0 & 0 \\
0 & -a^{t} & 0 \\
0 & 0 & 0
\end{array}\right), a \text { diagonal }\right\} .
$$

Then the irreducible representations of $\operatorname{SO}(k, \mathbb{R})$ are parametrized by the lowest weight $\left(m_{1}, \ldots, m_{1}\right)$, with $m_{1} \geqslant m_{2} \geqslant \cdots m_{1} \geqslant 0$. Thus, $\Sigma$ is parametrized by $\lambda=\left(m_{1}\right.$, $\left.\ldots, m_{1}, \varepsilon\right) . \varepsilon \in Z_{2}$ describes the representation of $Z_{2}$.

Let $x_{1}, \ldots, x_{l}, y_{1}, \ldots, y_{l}, t$ be the canonical basis of $\mathbb{C}^{k}$. The transformation from the natural bilinear form of $\mathbb{C}^{k}$ to $J$ can be chosen to be unitary and this basis becomes orthonormal. Thus, the commutation relations of the creation and annihilation operators are obvious. In this notation the representation $\mathrm{d} \tilde{\Gamma}$ of $\tilde{\Lambda}_{\text {res }}(V)$ on $\hat{S}_{k}$ 
transforms to

$$
\begin{aligned}
\mathrm{d} \tilde{\Gamma} & \left(\left(\begin{array}{cc}
a & b \\
c & -a^{t}
\end{array}\right), z\right) \\
= & \sum_{i, j}\left(a^{*}\left(v_{i} \otimes t\right) a\left(v_{j} \otimes t\right)+\sum_{\mu=1}^{l} a^{*}\left(v_{i} \otimes x_{\mu}\right) a\left(v_{j} \otimes x_{\mu}\right)+a^{*}\left(v_{i} \otimes y_{\mu}\right) a\left(v_{j} \otimes y_{\mu}\right)\right)\left\langle v_{i}, \mathbf{a v}_{j}\right\rangle \\
& +\sum_{i, j}\left(\frac{1}{2} a^{*}\left(v_{i} \otimes t\right) a^{*}\left(v_{i} \otimes t\right)+\sum_{\mu=1}^{l} a^{*}\left(v_{i} \otimes x_{\mu}\right) a^{*}\left(v_{j} \otimes y_{\mu}\right)\right)\left\langle v_{j}, b \bar{v}_{i}\right\rangle- \\
& -\sum_{i, j}\left(\frac{1}{2} a\left(v_{i} \otimes t\right) a\left(v_{j} \otimes t\right)+\sum_{\mu=1}^{l} a\left(v_{i} \otimes x_{\mu}\right) a\left(v_{j} \otimes y_{\mu}\right)\right)\left\langle\bar{v}_{j}, c v_{i}\right\rangle+\frac{z}{2} 1
\end{aligned}
$$

Let $e_{\mu, v} 1 \leqslant \mu, v \leqslant l$ be the canonical basis of $\mathrm{gl}(l, \mathbb{C}) . \mathrm{d} \tilde{\Gamma}$ is a representation of $\operatorname{so}(k, \mathbb{C})$ as a subalgebra of $\tilde{\Lambda}_{\text {res }}\left(V \otimes \mathbb{C}^{k}\right)$. Let $\mathrm{d} \Gamma$ denote the canonical representation of $\mathrm{gl}(k, \mathbb{C})$ on $S_{k}$, which is an extension of the first representation.

Set

$$
\begin{array}{lc}
A_{\mu, v}:=\mathrm{d} \Gamma\left(\left(\begin{array}{ccc}
e_{\mu, v} & 0 & 0 \\
0 & 0 & 0 \\
0 & 0 & 0
\end{array}\right)\right), & B_{\mu, v}:=\mathrm{d} \Gamma\left(\left(\begin{array}{ccc}
0 & e_{\mu, v} & 0 \\
0 & 0 & 0 \\
0 & 0 & 0
\end{array}\right)\right), \\
C_{\mu, v}:=\mathrm{d} \Gamma\left(\left(\begin{array}{ccc}
0 & 0 & 0 \\
e_{\mu, v} & 0 & 0 \\
0 & 0 & 0
\end{array}\right)\right), & D_{\mu, v}:=\mathrm{d} \Gamma\left(\left(\begin{array}{ccc}
0 & 0 & 0 \\
0 & e_{\mu, v} & 0 \\
0 & 0 & 0
\end{array}\right)\right), \\
E_{\mu}:=\mathrm{d} \Gamma\left(\left(\begin{array}{ccc}
0 & 0 & x_{\mu} \\
0 & 0 & 0 \\
0 & 0 & 0
\end{array}\right)\right), & F_{\mu}:=\mathrm{d} \Gamma\left(\left(\begin{array}{ccc}
0 & 0 & 0 \\
0 & 0 & y_{\mu} \\
0 & 0 & 0
\end{array}\right)\right),
\end{array}
$$

and

$$
H:=\mathrm{d} \Gamma\left(\left(\begin{array}{lll}
0 & 0 & 0 \\
0 & 0 & 0 \\
0 & 0 & 1
\end{array}\right)\right) .
$$

By definition, the following relations are valid:

$$
A_{\mu, v}^{*}=A_{v, \mu}, \quad D_{\mu, v}^{*}=D_{v, \mu}, \quad B_{v, \mu}^{*}=C_{\mu, v}, \quad H=H^{*} .
$$

Set

$$
\begin{aligned}
& \Delta_{\mu}(x):=\operatorname{det}\left(\begin{array}{ccc}
v_{1} \otimes x_{1} & \cdots & v_{1} \otimes x_{\mu} \\
\vdots & & \vdots \\
v_{\mu} \otimes x_{1} & \cdots & v_{\mu} \otimes x_{\mu}
\end{array}\right), \quad 1 \leqslant \mu \leqslant l
\end{aligned}
$$

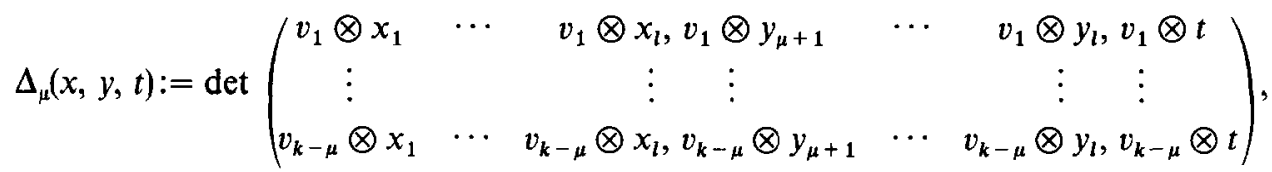


$0 \leqslant \mu \leqslant l$.

$$
\Delta_{1}^{\alpha_{1}}(x) \cdots \Delta_{\mu}^{\alpha_{\mu}}(x) \text { and } \Delta_{1}^{\alpha_{1}}(x) \cdots \Delta_{\mu}^{\alpha_{\mu}}(x) \Delta_{\mu}(x, y, t)
$$

are minimal weight vectors of $\hat{S}_{k}$. Their weights are $\left(\left\{m_{i}\right\}_{i \in \mathbb{N}}, n_{c}\right)$, with

$$
m_{i}=\left\{\begin{array}{l}
\Sigma_{j \geqslant i} \alpha_{j} \text { and } 1+\Sigma_{j \geqslant i} \alpha_{j}, \text { respectively, if } i \leqslant \mu, \\
0, \quad \text { if } i>\mu
\end{array}\right.
$$

and $n_{c}=k$.

In the case $k=2 l$, we choose the decomposition $\mathbb{C}^{k}=\mathbb{C}^{l} \oplus \mathbb{C}^{l}$ in order to express the elements of $\operatorname{gl}(k, \mathbb{C})$ and $\operatorname{so}(k, \mathbb{C})$. With

$$
J=\left(\begin{array}{ll}
0 & 1 \\
1 & 0
\end{array}\right)
$$

the elements of $\operatorname{so}(k, \mathbb{C})$ are of the form

$$
\left(\begin{array}{cc}
a & b \\
c & -a^{t}
\end{array}\right), \quad b, c \text { skew symmetric. }
$$

We take a Borel subalgebra

$$
b_{s o}^{-}:=\left\{\left(\begin{array}{ll}
a & b \\
0 & a^{t}
\end{array}\right), \quad a \text { upper triangular }\right\}
$$

and a Cartan subalgebra

$$
h_{s o}:=\left\{\left(\begin{array}{cc}
a & 0 \\
0 & -a^{t}
\end{array}\right), \quad a \text { diagonal }\right\} .
$$

an irreducible representation of $\operatorname{SO}(k, \mathbb{R})$ is parametrized by its lowest weight with respect to $b_{\text {so }}^{-}$, i.e. $\left(m_{1}, \ldots, m_{l}\right)$ with $m_{1} \geqslant \cdots \geqslant m_{l-1} \geqslant\left|m_{l}\right|$. In this case, $\mathrm{O}(k, \mathbb{R})$ is the semi-direct product of $\mathrm{SO}(k, \mathbb{R})$ and $Z_{2}$. In case $m_{l} \neq 0$ the representations $m_{l}=+-\left|m_{l}\right|$ of $\mathrm{SO}(k, \mathbb{R})$ induces only one irreducible representation of $\mathrm{O}(k, \mathbb{R})$. Otherwise it extends to two irreducible representations. Hence, $\Sigma$ is parametrized by

$$
\left(m_{1}, \ldots, m_{l}, \varepsilon\right), \quad m_{1} \geqslant \cdots \geqslant m_{l} \geqslant 0, \varepsilon \in Z_{2} \text { and }\left(m_{1}, \ldots, m_{l}, 1\right)=\left(m_{1}, \ldots, m_{l},-1\right)
$$
if $m_{l} \neq 0$.

Let $x_{1}, \ldots, x_{l}, y_{1}, \ldots, y_{l}$ be the canonical basis of $\mathbb{C}^{k}$, which is again orthonormal. In this notation the representation $\mathrm{d} \tilde{\Gamma}$ of $\tilde{\Lambda}_{\text {res }}(V)$ on $\hat{S}_{k}$ transforms to

$$
\begin{aligned}
\mathrm{d} \tilde{\Gamma} & \left(\left(\begin{array}{cc}
a & b \\
c & -a^{t}
\end{array}\right), z\right) \\
= & \sum_{i, j}\left(\sum_{\mu=1}^{l} a^{*}\left(v_{i} \otimes x_{\mu}\right) a\left(v_{j} \otimes x_{\mu}\right)+a^{*}\left(v_{i} \otimes y_{\mu}\right) a\left(v_{j} \otimes y_{\mu}\right)\right)\left\langle v_{i}, a v_{j}\right\rangle+ \\
& +\sum_{i, j}\left(\sum_{\mu=1}^{l} a^{*}\left(v_{i} \otimes x_{\mu}\right) a^{*}\left(v_{j} \otimes y_{\mu}\right)\right)\left\langle v_{j}, b \bar{v}_{i}\right\rangle- \\
& -\sum_{i, j}\left(\sum_{\mu=1}^{l} a\left(v_{i} \otimes x_{\mu}\right) a\left(v_{j} \otimes y_{\mu}\right)\right)\left\langle\bar{v}_{j}, c v_{i}\right\rangle+\frac{z}{2} 1 .
\end{aligned}
$$


Let $\mathrm{d} \Gamma$ denote the canonical representation of $\mathrm{g}(k, \mathbb{C})$ on $S_{k}$ which is an extension of the first representation. Set

$$
\begin{array}{ll}
A_{\mu, v}:=\mathrm{d} \Gamma\left(\left(\begin{array}{cc}
e_{\mu, v} & 0 \\
0 & 0
\end{array}\right)\right), & B_{\mu, v}:=\mathrm{d} \Gamma\left(\left(\begin{array}{cc}
0 & e_{\mu, v} \\
0 & 0
\end{array}\right)\right), \\
C_{\mu, v}:=\mathrm{d} \Gamma\left(\left(\begin{array}{cc}
0 & 0 \\
e_{\mu, v} & 0
\end{array}\right)\right), & D_{\mu, v}:=\mathrm{d} \Gamma\left(\left(\begin{array}{cc}
0 & 0 \\
0 & e_{\mu, v}
\end{array}\right)\right) .
\end{array}
$$

By definition the following relations are valid:

$$
A_{\mu, v}^{*}=A_{v, \mu}, \quad D_{\mu, v}^{*}=D_{v, \mu}, \quad B_{\mu, v}^{*}=C_{v, \mu} .
$$

Set

$$
\begin{aligned}
& \Delta_{\mu}(x):=\operatorname{det}\left(\begin{array}{ccc}
v_{1} \otimes x_{1} & \cdots & v_{1} \otimes x_{\mu} \\
\vdots & & \vdots \\
v_{\mu} \otimes x_{1} & \cdots & v_{\mu} \otimes x_{\mu}
\end{array}\right), \quad 1 \leqslant \mu \leqslant l,
\end{aligned}
$$

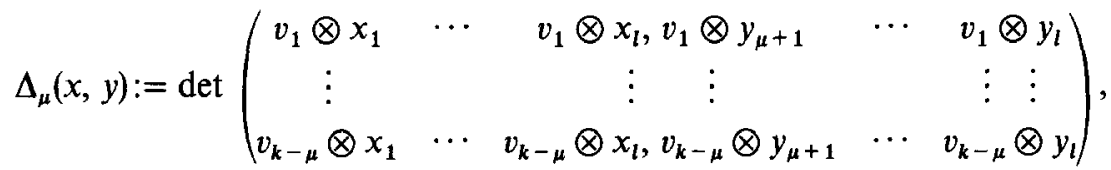

$0 \leqslant \mu \leqslant l$.

The elements $\Delta_{1}^{\alpha_{1}}(x) \cdots \Delta_{\mu}^{\alpha_{\mu}}(x)$ and $\Delta_{1}^{\alpha_{1}}(x) \cdots \Delta_{\mu}^{\alpha_{\mu}}(x) \Delta_{\mu}(x, y)$ are minimal weight vectors of $\hat{S}_{k}$. Their weights are $\left(\left\{m_{i}\right\}_{i \in \mathbb{N}}, n_{c}\right)$, with

$$
m_{i}=\left\{\begin{array}{l}
\Sigma_{j \geqslant i} \alpha_{j} \text { and } 1+\Sigma_{j \geqslant i} \alpha_{j}, \text { respectively, if } i \leqslant \mu, \\
0, \text { if } i>\mu,
\end{array}\right.
$$

and $n_{c}=k$. This leads to the following.

2.6. LEMMA. For all weights $\left(\left\{m_{i}\right\}_{i \in \mathbb{N}}, n_{\mathrm{c}}\right) \leqslant 0$, with $n_{\mathrm{c}} \geqslant \min \left\{i \mid n_{j}=0\right.$ for all $\left.j>i\right\}$ and $n_{c} / 2 \geqslant \min \left\{i \mid n_{j}=0,1\right.$ for all $\left.j>i\right\}$ there is a minimal weight vector in $\hat{S}_{n_{c}}$.

Kashiwara and Vergne [7] showed for finite dimensional $V$ that these are all minimal weight vectors and the representation of $\mathrm{Sp}(V)$ is completely reducible. Again we want to extend these results to the infinite dimensional case.

Let $b_{\mathrm{gl}}^{-}$be the Borel algebra of $\mathrm{gl}(k, \mathbb{C})$ of the form

$$
\left\{\left(\begin{array}{lll}
a & b & e \\
0 & d & 0 \\
0 & f^{*} & g
\end{array}\right), a \text { upper triangular, } d \text { downer triangular }\right\}
$$

and

$$
\left\{\left(\begin{array}{ll}
a & b \\
0 & d
\end{array}\right), a \text { upper triangular, } d \text { downer triangular }\right\},
$$

when $k$ is odd and even, respectively. 
2.7. LEMMA. All minimal weight vectors $v$ with respect to $b_{s o}^{-}$in $\hat{H}_{k}$, which corresponds to one $\lambda \in \Sigma$ are also minimal weight vectors with respect to $b_{\mathrm{gl}}^{-}$.

Proof. I only give the proof for $k$ odd and leave it to the reader to carry over the proof to the case $k$ even. For all $\mu=1, \ldots, l$

$$
\begin{aligned}
& -\sum_{i, j} a^{*}\left(v_{i} \otimes x_{v}\right) a^{*}\left(v_{j} \otimes y_{\mu}\right) \mathrm{d} \tilde{\Gamma}\left(\left(\begin{array}{cc}
0 & 0 \\
c_{i, j} & 0
\end{array}\right), 0\right) \\
& =\sum_{i, j} a^{*}\left(v_{j} \otimes y_{\mu}\right) a\left(v_{j} \otimes t\right) a^{*}\left(v_{i} \otimes x_{\mu}\right) a\left(v_{i} \otimes t\right)+ \\
& \quad+\sum_{i, j, v} a^{*}\left(v_{j} \otimes y_{\mu}\right) a\left(v_{j} \otimes y_{v}\right) a^{*}\left(v_{i} \otimes x_{\mu}\right) a\left(v_{i} \otimes x_{v}\right)+ \\
& \quad+\sum_{i, j, v} a^{*}\left(v_{j} \otimes y_{\mu}\right) a\left(v_{j} \otimes x_{v}\right) a^{*}\left(v_{i} \otimes x_{\mu}\right) a\left(v_{i} \otimes y_{v}\right)- \\
& \quad-\sum_{i, j, v} a^{*}\left(v_{j} \otimes y_{\mu}\right)\left[a\left(v_{j} \otimes x_{v}\right), a^{*}\left(v_{i} \otimes x_{\mu}\right)\right] a\left(v_{i} \otimes y_{v}\right) \\
& =F_{\mu} E_{\mu}-D_{\mu, \mu}+\sum_{\nu=1}^{l} D_{\mu, v} A_{\mu, v}+C_{\mu, v} B_{\mu, v} .
\end{aligned}
$$

By definition, $\hat{H}_{k}$ has to be a subset of the kernel of all these operators. Now let $v$ be a minimal weight with respect to $b_{s o}^{-}$with weight $\left(m_{1}, \ldots, m_{l}\right)$. This implies

$$
\begin{aligned}
& \left(E_{\mu}-F_{\mu}^{*}\right) v=0 \text { for all } \mu=1, \ldots, l, \\
& \left(A_{\mu, \nu}-D_{v, \mu}\right) v=0 \text { for all } 1 \leqslant \mu<v \leqslant l, \\
& \left(B_{\mu, \nu}-B_{v, \mu}\right) v=0 \text { for all } \mu, v
\end{aligned}
$$

and

$$
\left(A_{\mu, \mu}-D_{\mu, \mu}\right) v=m_{v} v .
$$

Hence, $v$ must be in the kernel of

$$
D_{1,1}\left(A_{1,1}-1\right)+F_{1} F_{1}^{*}+B_{1,1}^{*} B_{1,1}+\sum_{v=2}^{l} D_{1, v} D_{1, v}^{*}+B_{v, 1}^{*} B_{v, 1} .
$$

This implies $\left\langle v, D_{1,1}\left(A_{1,1}-1\right) v\right\rangle \leqslant 0$. If $m_{1} \geqslant 1$ this condition implies $A_{1,1} v=m_{1} v$, because $A_{1,1}$ and $D_{1,1}$ are positive. If $m_{1}=0$ there are two possibilities:

$$
A_{1,1} v=0 \text { and } D_{1,1} v=0 \text { or } A_{1,1} v=v \text { and } D_{1,1} v=\mathrm{v} \text {. }
$$

This implies

$$
\begin{aligned}
& D_{1,1}\left(A_{1,1}-1\right) v=0 \text { and } E_{1} v=F_{1}^{*} v=0 \\
& A_{1, v} v=D_{v, 1} v=0 \text { for } v>1 \text { and } B_{1, \mu} v=B_{\mu, 1} v=0
\end{aligned}
$$

Using this methods iteratively for $\mu=1, \ldots, l$, one finally gets

$$
\begin{aligned}
& D_{\mu, \mu}\left(A_{\mu, \mu}-1\right) v=0, \quad E_{\mu} v=F_{\mu}^{*} v=0, \\
& A_{\mu, v} v=D_{v, \mu} v=0 \quad \text { for all } \mu<v, \quad \text { and } \quad B_{\mu, v} v=0 \text { for all } \mu, v .
\end{aligned}
$$


This implies that $v$ is the sum of minimal weight vectors with respect to $b_{\mathrm{gl}}^{-}$. Now let $v$ be such a minimal weight vector with weight $\left(a_{1}, \ldots, a_{l}, d_{1}, \ldots, d_{l}, h\right)$. These nonnegative integers must obey the following inequalities: $a_{1} \geqslant \cdots \geqslant a_{l} \geqslant h \geqslant d_{l} \geqslant \cdots \geqslant d_{1} \geqslant 0$, since they represent a minimal weight of a unitary representation of $U(k, \mathbb{C})$. Now we have

$$
\begin{aligned}
& -\sum_{i, j} a^{*}\left(v_{i} \otimes t\right) a^{*}\left(v_{j} \otimes t\right) \mathrm{d} \tilde{\Gamma}\left(\left(\begin{array}{cc}
0 & 0 \\
c_{i, j} & 0
\end{array}\right), 0\right) \\
& =\sum_{i, j} a^{*}\left(v_{i} \otimes t\right) a\left(v_{i} \otimes t\right) a^{*}\left(v_{j} \otimes t\right) a\left(v_{j} \otimes t\right)- \\
& \quad-\sum_{i, j} a^{*}\left(v_{i} \otimes t\right)\left[a\left(v_{i} \otimes t\right), a^{*}\left(v_{j} \otimes t\right)\right] a\left(v_{j} \otimes t\right)+ \\
& \quad+2 \sum_{i, j, \mu} a^{*}\left(v_{i} \otimes t\right) a\left(v_{i} \otimes x_{\mu}\right) a^{*}\left(v_{j} \otimes t\right) a\left(v_{j} \otimes y_{\mu}\right) \\
& =H(H-1)+2 \sum_{\mu=1}^{l} E_{\mu}^{*} F_{\mu}^{*} .
\end{aligned}
$$

So $v$ lies also in the kernel of this operator and there are only two cases

(1) $h=0$ : This implies $d_{v}=0$ and $a_{v}=m_{v}$.

(2) $h=1$ : Then $d_{v}\left(a_{v}-1\right)=0$ implies

$$
d_{v}=\left\{\begin{array}{ll}
0, & \text { if } m_{v} \geqslant 1 \\
1, & \text { if } m_{v}=0
\end{array}, \quad a_{v}= \begin{cases}m_{v}, & \text { if } m_{v} \geqslant 1 \\
1, & \text { if } m_{v}=0\end{cases}\right.
$$

In case $k$ is even, the second case only occurs if $m_{l}=0$. These two minimal weights corresponds to different representations of $\mathrm{O}(k, \mathbb{R})$, because $-1 \in \mathrm{O}(k, \mathbb{R})$ is represented differently. This concludes the proof.

2.8. COROLLARY. For all $\lambda \in \Sigma$, there is at most one $n \in \mathbb{N}$ such that $L_{\lambda}\left(H_{k}^{n}\right) \neq 0$. $L_{\lambda}\left(\hat{H}_{k}\right)$ is an irreducible representation of $C(V)$.

Proof. The preceding lemma implies the first statement. $S_{k}^{n}$ is of course an irreducible representation of $\mathrm{gl}(k \mathbb{C}) \times C(V)$. Hence, the preceding lemma implies also the second statement.

2.9. PROPOSITION. $L_{\lambda}\left(\hat{S}_{k}\right)$ is an irreducible representation of $\tilde{\Lambda}_{\mathrm{res}}(V) . L_{\lambda}\left(S_{k}\right)$ is an irreducible unitary representation of $\tilde{\mathrm{S}}_{\mathrm{res}}(V)$.

Proof. Set

$$
N:=\mathrm{d} \tilde{\Gamma}\left(\left(\begin{array}{rr}
1 & 0 \\
0 & -1
\end{array}\right), 0\right)
$$

Each closed subspace of $\hat{S_{k}}$, which is invariant with respect to $N$ contains its orthogonal components in $S_{k}^{n}$. So every invariant subspace of $L_{\lambda}\left(\hat{S}_{k}\right)$ meets $L_{\lambda}\left(\hat{H}_{k}\right)$, because all operators of the form

$$
\mathrm{d} \tilde{\Gamma}\left(\left(\begin{array}{ll}
0 & 0 \\
c & 0
\end{array}\right), 0\right)
$$


reduce $n$ by two. Due to the preceding lemma it then must contain this space. By the unitarity of the representation of $\tilde{S}_{\text {res }}(V)$ on $L_{\lambda}\left(S_{k}\right)$ it now follows that $L_{\lambda}\left(\hat{S}_{k}\right)$ is an irreducible representation of $\tilde{\Lambda}_{\text {res }}(V)$.

2.10. THEOREM. $S_{k}$ is completely reducible in unitary lowest weight representations of $\tilde{\mathrm{S}}_{\mathrm{res}}(V)$. The decomposition is explicitly given by

$$
S_{k}=\bigoplus_{\lambda \in \Sigma}\left(\bar{V}_{\lambda} \otimes L_{\lambda}\left(S_{k}\right) \quad\right. \text { (completion of the infinite sum). }
$$

The $L_{\lambda}\left(S_{k}\right)$ are all distinct.

Proof. It remains to show that the $L_{\lambda}\left(S_{k}\right)$ are lowest weight representations and are all distinct. If $\lambda=\left(m_{1}, \ldots, m_{l},(-1) \Sigma^{m_{\mu}}\right)$ the vector $\Delta_{1}^{m_{1}-m_{2}}(x) \Delta_{2}^{m_{2}-m_{3}}(x) \cdots \Delta_{l}^{m_{1}}(x)$ is a minimal weight vector with respect to $b_{\mathrm{gl}}^{-}$and $\tilde{b}_{\text {res }}^{-}(V)$ which corresponds to $\lambda$. The corresponding weight is $\left(\left(m_{1}, \ldots, m_{l}, 0, \ldots\right), k\right)$.

If $\lambda=\left(m_{1}, \ldots, m_{l},(-1)^{1+\Sigma m_{\mu}}\right)$, let $\mu$ be the greatest integer, such that $m_{\mu} \neq 0$. Then

$$
\Delta_{1}^{m_{1}-m_{2}}(x) \Delta_{2}^{m_{2}-m_{3}}(x) \cdots \Delta_{\mu}^{m_{\mu}-1}(x) \Delta_{\mu}(x, y)
$$

and

$$
\Delta_{1}^{m_{1}-m_{2}}(x) \Delta_{2}^{m_{2}-m_{3}}(x) \cdots \Delta_{\mu}^{m_{\mu}-1}(x) \Delta_{\mu}(x, y, t),
$$

respectively, are minimal weight vectors with respect to $b_{\mathrm{g} !}^{-}$and $\tilde{b}_{\text {res }}^{-}(V)$, which corresponds to $\lambda$. The corresponding weight of $\tilde{\Lambda}_{\text {res }}(V)$ is

$$
\left(\left(\underset{\leftarrow}{m_{1}}, \ldots, \underset{k-j}{m_{j}}, 1, \ldots, 1,0, \ldots\right), k\right) .
$$

2.11. Remark. Enright and Parthasarathy [9] et al. [11-10] showed that all unitary lowest weight representations of $\operatorname{Sp}(V)$ are contained in $S_{k}$ for some $k$, if $V$ is finite dimensional. If $V$ is not finite dimensional in all weights $\left(\left\{\boldsymbol{m}_{i}\right\}_{i \in \mathbb{N}}, n_{c}\right)$, only a finite number of the $m_{i}$ 's are different from zero. The embeddings of finite dimensional groups $\mathrm{Sp}\left(V^{\prime}\right)$ into $\widetilde{\mathrm{Sp}}_{\mathrm{pes}}(V)$ then guarantee that all unitary lowest weight representations of $\widetilde{S}_{\text {res }}(V)$ are contained in some $S_{k}$.

\section{Acknowledgement}

I would like to thank R. Schrader and S. N. M. Ruijsenaars for discussion and help.

\section{References}

1. Segal, G. B.: Unitary representations of some infinite dimensional groups, Commun. Math. Phys. 80 (1981), 301-342.

2. Carey, A. L. and Ruijsenaars, S. N. M.: On fermion gauge groups, current algebras and Kac-Moody algebras, Acta Appl. Math. 10 (1987), 1-86.

3. Shale, D.: Linear symmetries of free boson fields, Trans. Amer. Math. Soc. 103 (1962), 149-167.

4. Schroer, B., Seiler, R., and Swieca, J.: Problems of stability for quantum fieids in external timedependent potentials, Phys. Rev. D2 (1970), 2927-2937. 
5. Ruijsenaars, S. N. M.: On Bogoliubov transformations II, Ann. of Phys. 116 (1978), 105-134.

6. Macdonald, I. G.: Symmetric Functions and Hall Polynomials, Oxford University Press, Oxford, 1979.

7. Kashiwara, M. and Vergne, M.: On the Segal-Shale-Weil representations and harmonic polynomials, Invent. Math. 44 (1978), 1-47.

8. Jakobsen, H.: On singular holomorphic representations, Invent. Math. 62 (1980), 67-78.

9. Enright, T. and Parthasarathy, R.: A proof of a conjecture of Kashiwara and Vergne, in J. Carmona and M. Vergne, Non Commutative Harmonic Analysis and Lie Groups, Lecture Notes in Mathematics 880, Springer-Verlag, Berlin, 1981, pp. 74-90.

10. Enright, T., Howe, R., and Wallach, N.: A classification of unitary highest weight modules, in P. C. Trombi (ed.), Representation Theory of Reductive Groups, Birkhäuser-Verlag, Boston, 1983, pp. 97-143.

11. Jakobsen, $H$. .: The last possible place of unitarity for certain highest weight modules, Math. Ann. 256 (1981), 439-447.

12. Jakobsen, H.: Hermitian symmetric spaces and their unitary highest weight modules, J. Funct. Anal. 52 (1983), 385-412. 